\title{
Multiple scattering theory of proton elastic scattering at intermediate energies
}

\author{
R. Crespo, ${ }^{*}$ R. C. Johnson, and J. A. Tostevin \\ Department of Physics, University of Surrey, Guildford, Surrey, GU2 5XH, United Kingdom
}

(Received 14 November 1991)

\begin{abstract}
We derive and calculate the multiple scattering expansion of the Kerman-McManus-Thaler optical potential to second order in the free nucleon-nucleon transition amplitude and clarify the physical content of the interaction. Calculations are presented for the elastic scattering of protons from ${ }^{16} \mathrm{O}$ at 135 , 200 , and $300 \mathrm{MeV}$ incident energies, paying particular attention to the nonlocalities inherent in the second-order potential. It is shown that these effects result in a significant reduction in the proton-target absorption.
\end{abstract}

PACS number(s): 24.10. $-\mathrm{i}, 24.10 . \mathrm{Ht}, 24.70 .+\mathrm{s}, 25.40 . \mathrm{Cm}$

\section{INTRODUCTION}

A large number of very precise data on proton-nucleus elastic and inelastic scattering have recently been obtained using intermediate energy beams [1]. In addition, a number of important high-energy experiments have been carried out or are planned with weak probes, for example, $\left(e, e^{\prime} p\right)$ reactions [2]. Whereas in elastic scattering the reaction is surface dominated the $\left(p, p^{\prime}\right)$ and $\left(e, e^{\prime} p\right)$ reactions are sensitive to the proton-nucleus wave function within the target nucleus. It is timely therefore to study quantitatively the proton-nucleus optical potential in the nuclear interior as well as at the nuclear surface.

In parallel with these experimental developments, recent investigations of the free nucleon-nucleon $(N N)$ transition amplitude calculated from realistic $N N$ interactions [3] have shown the on- and off-shell behavior of the amplitude to be remarkably stable to the choice of interaction [4]. This, combined with more detailed information on nuclear wave functions now available from electron scattering data, has stimulated a renewed interest in finite nucleus calculations of the nucleon-nucleus $(N A)$ interaction based on the free $N N$ transition amplitude and multiple scattering expansions, as formulated, for example, by Kerman, McManus, and Thaler (KMT) [5]. From the preceeding discussion, the KMT optical potential is expected to be relatively insensitive to the assumed $N N$ interaction. Since the on-shell $N N$ amplitude is constrained by the experimental phase shifts it follows that the $N A$ optical potential should be fairly well determined by empirical $N N$ information, at least on the energy shell. This approach builds in automatically, on the energy shell, the subtle cancellations arising from the attractive and repulsive contributions of the $N N$ interaction in each angular momentum state.

The second most often used procedure of generating the microscopic $N N$ information required as input to the $N A$ optical potential is to construct the $N N g$-matrix effective interaction appropriate for nuclear matter [6]. Several groups have evaluated the $g$ matrix, for a variety

\footnotetext{
*On leave from Departamento de Física, Instituto Superior Técnico, Lisboa, Portugal.
}

of $N N$ potentials and approximation strategies [7-9]. The validity of these approximations has been discussed in the literature [10]. In this approach the local density approximation has to be used in applying the nuclear matter results to finite nuclei. This approximation is suspect [9] for two reasons. Firstly, in processes such as elastic scattering which are surface dominated the procedure is ill defined due to rapid variations in the spatial density distribution. Secondly, the approach involves an approximate solution of the $g$-matrix equation which introduces approximations to the direct and exchange parts of the amplitude and to the short-ranged repulsion and longranged attraction of the $N N$ effective interaction. These approximations compromise the delicate cancellations between these terms referred to above. In addition, or as a result of the above, $g$-matrix calculations have been found to exhibit considerable sensitivity to the adopted $N N$ potential model $[9,11]$.

The first-order term of the KMT nucleon-nucleus potential will be referred to as the single scattering approximation (SSA). Given by the expectation value of the free $N N$ transition amplitude, in the target nucleus ground state, the SSA is expected, at intermediate energies, to generate most of the scattering from the nucleus. The off-energy shell effects of the $N N$ transition amplitude, which arise because the projectile scatters from a bound nucleon in the $N A$ center-of-mass system, have recently been the subject of a number of very detailed investigations [12-14] and will not be covered in detail here. Clearly, however, the significance of any agreement or disagreement of these SSA calculations with experimental data is impossible to assess without realistic calculations of the importance of higher order and in particular second-order contributions to the $N A$ interaction. In this paper, we address this problem. We present the most complete calculations to date of the second-order term of the nucleon-nucleus potential. We will pay particular attention to the effects of the nonlocalities, inherent in the potential, on the absorptive nature of the optical potential. We derive the KMT optical potential to second order in the free $N N$ transition amplitude, assuming that the target ground state is described by a Slater determinant of occupied single particle states. The proton $-{ }^{16} \mathrm{O}$ optical potential is calculated, in momentum space repre- 
sentation, at 135,200 , and $300 \mathrm{MeV}$ incident energies using both harmonic oscillator and Woods-Saxon single particle wave functions. The free $N N$ transition amplitude is that of the Paris potential [15]. In the present work the spin-orbit component of the second-order $N A$ potential is neglected. An estimate of the importance of this term for polarization data was made recently by Feshbach [16].

The second-order term of the multiple scattering expansion to the $N A$ potential involves a "double scattering" between the incident and target nucleons and will be referred to as the DSA. We show that this term modifies the free $N N$ transition amplitude, appearing in the SSA term, to account for Pauli blocking medium effects due to the identity of the target nucleons. The evaluation of the DSA terms requires a knowledge of the two particle correlations in the target nucleus and a complete description of the spin and isospin dependence of the free $N N$ amplitude. In this work we consider only Pauli correlations.

A basic problem with the DSA potential is its nonlocality which originates from two sources. Firstly, as in the SSA, nonlocality arises through the $N N$ transition amplitude. In addition, nonlocality arises from the propagation of the nucleons in the intermediate state between scattering events. It is the role and importance of these two sources of nonlocality which will be clarified in this paper. Due to the complexity of the nonlocal secondorder potential, previous calculations have attempted to localize it using the eikonal approximation to the propagator, simple models for the target correlation function, e.g., that deduced from a Fermi gas, and approximate prescriptions for the $N N$ transition amplitude [17]. Two analyses, by Fesbach et al. [18] and Johnson and Martin [19], did include the nonlocality associated with the intermediate states propagator. Both analyses reduce the evaluation of the DSA terms to the solution of a finite set of coupled equations. Neither approach however included fully the folding of the nonlocal $N N$ transition amplitude with the target wave function. These effects are included explicitly in the present work.

Very recently detailed calculations of proton scattering, and a discussion of off-shell effects, have been presented by Ottenstein et al. [20] within the relativistic (Dirac equation) SSA optical potential formulation. Second order medium effects in the relativistic framework have also been evaluated by Kaki [21]. The relativistic approach includes virtual pair contributions in addition to the dynamics incorporated in the nonrelativistic calculations. It will be important to reconcile the relative importance of off-shell and medium effects obtained using the two approaches [22].

\section{THE OPTICAL POTENTIAL}

\section{A. The KMT multiple scattering expansion}

In the KMT multiple scattering formalism [5] the antisymmetrization of the projectile with the struck nucleon and of the struck nucleon with the remaining $A-1$ target nucleons is properly taken into account. No attempt is made however to consider the full antisymmetrization of the projectile with the latter $(A-1)$ spectator nucleons. Thus one neglects target exchange effects in which a target nucleon other than the struck nucleon is removed from the target and the incident nucleon remains in the nucleus. Such contributions, which involve the overlap of nucleon bound and scattering states, can be neglected at intermediate energies [23,24].

The optical potential operator for the scattering of a nucleon with energy $E$ from a target of mass $A$ is written [5]

$$
U=(A-1) \tau_{01}(E)\left[1+\frac{\mathcal{A}}{\delta} Q_{0} U\right]
$$

Here $\mathcal{A}$ is the antisymmetrization operator for the $A$ target nucleons and $\delta=E^{+}-K_{0}-H_{A}$ with $E=\hbar^{2} k_{0}^{2} / 2 \mu_{N A}$ and $\mu_{N A}$ the $N A$ reduced mass. $H_{A}$ is the internal Hamiltonian of the target, $K_{0}$ the kinetic energy operator of the incident nucleon, and $k_{0}$ its on-shell $N A$ momentum. The Pauli blocking operator $Q_{0}$ projects off the target ground state $\phi_{0}$, i.e., $Q_{0}=1-P_{0}$ where $P_{0}=\left|\phi_{0}\right\rangle\left\langle\phi_{0}\right|$. The antisymmetrized effective $N N$ transition operator $\tau_{01}(E)$, which describes the scattering of the projectile with any one of the target nucleons (labelled " 1 "), satisfies the integral equation

$$
\tau_{01}(E)=v_{01}+v_{01} \frac{\mathcal{A}}{\delta} \tau_{01}(E)
$$

The presence of the antisymmetrization operator allows only physical states of the nucleus as intermediate states. Upon expanding the integral equation for the optical potential operator, Eq. (1), to second order in $\tau_{01}$ we thus obtain the second-order optical potential for elastic scattering

$$
\begin{aligned}
U^{\mathrm{opt}}= & (A-1)\left\langle\phi_{0}\left|\tau_{01}(E)\right| \phi_{0}\right\rangle \\
& +(A-1)^{2}\left\langle\phi_{0}\left|\tau_{01}(E) \frac{\mathcal{A}}{\delta} Q_{0} \tau_{01}(E)\right| \phi_{0}\right\rangle .
\end{aligned}
$$

We now introduce the $N N$ transition operator

$$
t_{01}(E)=v_{01}+v_{01} \frac{1}{\delta} t_{01}(E)
$$

which is not that describing free $N N$ scattering since the propagator remains a many-body operator. The unit partition in the propagator refers to the full Hilbert space of the target nucleus and includes both symmetric and antisymmetric states of the target nucleons. The relationship of $\tau_{01}$ to $t_{01}$ is, from Eq. (2),

$$
\tau_{01}(E)=t_{01}(E)+t_{01}(E) \frac{\mathcal{A}-1}{\delta} \tau_{01}(E)
$$

and, substituting this expression for $\tau_{01}$ in the optical potential, and retaining terms to second order in $t_{01}$ then

$$
\begin{aligned}
U^{\mathrm{opt}}= & (A-1)\left\langle\phi_{0}\left|t_{01}(E)\right| \phi_{0}\right\rangle \\
& +(A-1)\left\langle\phi_{0}\left|t_{01}(E) \frac{\mathcal{A}-1}{\delta} t_{01}(E)\right| \phi_{0}\right\rangle \\
& +(A-1)^{2}\left\langle\phi_{0}\left|t_{01}(E) \frac{\mathcal{A}}{\delta} Q_{0} t_{01}(E)\right| \phi_{0}\right\rangle,
\end{aligned}
$$


which can be reorganized, using $P_{0}+Q_{0}=1$, to give

$$
\begin{aligned}
U^{\mathrm{opt}}= & (A-1)\left\langle\phi_{0}\left|t_{01}(E)\right| \phi_{0}\right\rangle \\
& -(A-1)^{2}\left\langle\phi_{0}\left|t_{01}(E) \frac{P_{0}}{\delta_{0}} t_{01}(E)\right| \phi_{0}\right\rangle \\
& +\frac{A-1}{A} \sum_{i \neq j}\left\langle\phi_{0}\left|t_{0 i}(E) \frac{1}{\delta} t_{0 j}(E)\right| \phi_{0}\right\rangle,
\end{aligned}
$$

with $\delta_{0}=E^{+}-K_{0}$.

If the target ground state wave function is assumed to be a single Slater determinant of occupied single particle states $|\alpha\rangle$, with single particle energies $\epsilon_{\alpha}$, then (see Appendix $A$ for details) the optical potential reduces to $U^{\text {opt }}=U^{(1)}+U^{(2)}$, where the first-order term $U^{(1)}$ is

$$
U^{(1)}=\frac{A-1}{A} \sum_{\alpha}\left\langle\alpha\left|t_{01}^{f}\left(\hat{\omega}_{\alpha}\right)\right| \alpha\right\rangle
$$

and the second-order term $U^{(2)}$ is the sum of two components, $U^{(2)}=U_{I}^{(2)}+U_{I I}^{(2)}$, where

$$
\begin{aligned}
U_{I}^{(2)}=-\frac{A-1}{A} \sum_{\alpha, \beta}\left\langle\alpha\left|t_{01}^{f}\left(\hat{\omega}_{\beta}\right)\right| \beta\right\rangle & \\
& \times \frac{1}{E^{+}-K_{0}+\epsilon_{\alpha}-\epsilon_{\beta}}\left\langle\beta\left|t_{01}^{f}\left(\hat{\omega}_{\alpha}\right)\right| \alpha\right\rangle,
\end{aligned}
$$

$U_{I I}^{(2)}=\frac{A-1}{A^{2}} \sum_{\alpha, \beta}\left\langle\alpha\left|t_{01}^{f}\left(\hat{\omega}_{\alpha}\right)\right| \alpha\right\rangle \frac{1}{E^{+}-K_{0}}\left\langle\beta\left|t_{01}^{f}\left(\widehat{\omega}_{\beta}\right)\right| \beta\right\rangle$,

the sums in $\alpha$ and $\beta$ running over all occupied states. In Eqs. (8)-(10) $t_{01}^{f}\left(\hat{\omega}_{\alpha}\right)$ is now the $N N$ transition operator for energy parameter $\widehat{\omega}_{\alpha}$ which satisfies the integral equation,

$$
t_{01}^{f}\left(\widehat{\omega}_{\alpha}\right)=v_{01}+v_{01} \frac{1}{\hat{\omega}_{\alpha}^{+}-K} t_{01}^{f}\left(\widehat{\omega}_{\alpha}\right),
$$

where $K$ is the $N N$ relative kinetic energy and the energy parameter is, according to Eq. (A11) of Appendix A,

$$
\widehat{\omega}_{\alpha}=E+\epsilon_{\alpha}-\frac{\hbar^{2} \hat{\mathcal{P}}^{2}}{4 m}+K_{1}-h_{1} \text {. }
$$

In this equation $h_{1}$ is the single particle Hamiltonian of the struck nucleon, $\hat{P}$ the momentum operator for the center-of-mass motion of the interacting $N N$ pair, and $m$ is the nucleon mass.

The formulation of the KMT multiple scattering expansion above does not include the effects of antisymmetrization of the projectile and struck nucleon. These effects are taken into account by using a properly antisymmetrized free $N N$ transition amplitude.

\section{B. The impulse approximation}

The formulas for the first- and second-order optical potentials involve momentum space matrix elements of the form

$$
\begin{aligned}
\left\langle\mathbf{k}^{\prime}\left|\mathcal{U}_{\beta \alpha}\right| \mathbf{k}\right\rangle & =\left\langle\mathbf{k}^{\prime} \beta\left|t_{01}^{f}\left(\widehat{\omega}_{\alpha}\right)\right| \mathbf{k} \alpha\right\rangle \\
& =\int d \mathbf{p} \int d \mathbf{p}^{\prime}\left\langle\beta \mid \mathbf{p}^{\prime}\right\rangle\left\langle\mathbf{k}^{\prime} \mathbf{p}^{\prime}\left|t_{01}^{f}\left(\hat{\omega}_{\alpha}\right)\right| \mathbf{k} \mathbf{p}\right\rangle\langle\mathbf{p} \mid \alpha\rangle
\end{aligned}
$$

The effective operator $t_{01}^{f}\left(\hat{\omega}_{\alpha}\right)$, with energy parameter defined by Eq. (12), is still a three-body operator due to the presence of the struck nucleon-core binding potential $V_{1}=h_{1}-K_{1}$. This potential is typically of order -40 $\mathrm{MeV}$. To allow an unambiguous reduction to a two-body operator it is usual at intermediate energies to assume the impulse approximation such that, for the evaluation of $\widehat{\omega}_{\alpha}$, the projectile is assumed to scatter from a free nucleon. That is, we assume that the incident nucleon energy is sufficiently large that the single particle energy $\epsilon_{\alpha}$ and the nucleon-core potential $V_{1}$ of the struck nucleon can be neglected $[13,14]$. The medium effects arising from the distortion generated by $V_{1}$ in the intermediate states propagator are thus not considered.

Using momentum conservation for the interacting nucleon pair now gives

$$
\begin{aligned}
\left\langle\mathbf{k}^{\prime} \mathbf{p}^{\prime}\left|t_{01}^{f}(\omega)\right| \mathbf{k} \mathbf{p}\right\rangle & =\delta\left(\mathbf{k}^{\prime}+\mathbf{p}^{\prime}-\mathbf{k}-\mathbf{p}\right) \\
& \times\left\langle\frac{\mathbf{k}^{\prime}-\mathbf{p}^{\prime}}{2}\left|t_{01}^{f}(\omega)\right| \frac{\mathbf{k}-\mathbf{p}}{2}\right\rangle \\
& =\delta\left(\mathscr{P}^{\prime}-\mathcal{P}\right)\left\langle\mathcal{K}^{\prime}\left|t_{01}^{f}(\omega)\right| \mathcal{K}\right\rangle
\end{aligned}
$$

with

$$
\omega=E-\frac{\hbar^{2} \mathcal{P}^{2}}{4 m}=E-\frac{\hbar^{2}|\mathbf{k}+\mathbf{p}|^{2}}{4 m},
$$

in which the initial and final relative momenta of the two active nucleons are denoted by $\mathcal{K}$ and $\mathcal{K}^{\prime}$ and the momentum transfer by $\mathbf{q}=\mathbf{k}^{\prime}-\mathbf{k}=\mathcal{K}^{\prime}-\mathcal{K}$. The second term in the energy parameter accounts for the recoil of the target nucleus.

Since $t_{01}^{f}(\omega)$ is a slowly varying function of energy [25], for the evaluation of $\omega$ we also neglect the momentum $p$ of the struck nucleon compared to the incident nucleon momentum and take for $k$ its on-shell value $k_{0}=\sqrt{2 m E / \hbar^{2}}$. It then follows that the required $N N$ transition amplitude should be evaluated at a fixed energy $\omega=E / 2$, corresponding to free $N N$ scattering at half the beam energy in the $N N$ center-of-mass frame $[13,14]$. This is the approximation used throughout this paper. Hence nonlocalities arising from the energy dependence of $t^{f}(\omega)$ are not considered.

In Ref. [12] an attempt was made to include the nonlocalities associated with the center-of-mass momentum of the active pair $\mathcal{P}$. A correction to the impulse approximation was also made by including the single particle energy $\epsilon_{\alpha}$. Since the resulting calculations were affected significantly by these inclusions a full treatment of the binding corrections, including the effects of the distorting potential $V_{1}$ is needed to clarify the accuracy of the $\omega=E / 2$ prescription.

\section{The physical content}

In the KMT formalism the elastic scattering observables are calculated in terms of the $N A$ transition ampli- 
tude $T^{\mathrm{KMT}}=A /(A-1) T(U)$, where $T(U)$ is the transition operator generated from the optical potential $U$ of the previous section. Thus, to second order in $t_{01}^{f}$, we can write using Eqs. (8) $-(10), U=(A-1) \gamma$ where

$$
\begin{aligned}
\gamma= & \frac{1}{A} \sum_{\alpha}\left\langle\alpha\left|t_{01}^{f}\left(\widehat{\omega}_{\alpha}\right)\right| \alpha\right\rangle \\
& -\frac{1}{A} \sum_{\alpha, \beta}\left\langle\alpha\left|t_{01}^{f}\left(\widehat{\omega}_{\beta}\right)\right| \beta\right\rangle \frac{1}{\delta_{\alpha \beta}}\left\langle\beta\left|t_{01}^{f}\left(\widehat{\omega}_{\alpha}\right)\right| \alpha\right\rangle \\
& +\frac{1}{A^{2}} \sum_{\alpha, \beta}\left\langle\alpha\left|t_{01}^{f}\left(\hat{\omega}_{\alpha}\right)\right| \alpha\right\rangle \frac{1}{\delta_{0}}\left\langle\beta\left|t_{01}^{f}\left(\hat{\omega}_{\beta}\right)\right| \beta\right\rangle,
\end{aligned}
$$

with $\delta_{\alpha \beta}=E^{+}-K_{0}+\epsilon_{\alpha}-\epsilon_{\beta}$. It can also be shown [5] that the $N A$ transition amplitude satisfies the exact relationship

$$
T(A \Gamma)=\frac{A}{A-1} T([A-1] \gamma),
$$

if

$$
\Gamma=\gamma-\Gamma \frac{1}{\delta_{0}} \gamma=\gamma-\gamma \frac{1}{\delta_{0}} \gamma+\mathcal{O}\left(\gamma^{3}\right)+\cdots .
$$

Equation (17) shows that the physical scattering amplitude is generated by the potential $A \Gamma$. Substituting in this expression for $\gamma$ from Eq. (16) and retaining terms to second order in the $N N$ amplitude, the term of second order in $\gamma$ exactly cancels the third term of Eq. (16) and gives

$$
\begin{aligned}
A \Gamma= & \sum_{\alpha}\left\langle\alpha\left|t_{01}^{f}\left(\hat{\omega}_{\alpha}\right)\right| \alpha\right\rangle \\
& -\sum_{\alpha, \beta}\left\langle\alpha\left|t_{01}^{f}\left(\hat{\omega}_{\beta}\right)\right| \beta\right\rangle \frac{1}{\delta_{\alpha \beta}}\left\langle\beta\left|t_{01}^{f}\left(\hat{\omega}_{\alpha}\right)\right| \alpha\right\rangle+\mathcal{O}\left(t^{3}\right) .
\end{aligned}
$$

Thus the term $U_{I I}^{(2)}$, Eq. (10), which arises naturally in the KMT expansion for the second-order optical potential, has to be included to account correctly for all contributions from target ground state matrix elements of the $N N$ transition amplitude to the physical scattering amplitude.

The meaning of the second term in Eq. (19) is clear. Its role is to subtract off contributions from the first-order term from collisions between particles 0 and 1 which produce an intermediate state with particle 1 in an occupied state and which are therefore forbidden by the Pauli principle.

We now discuss briefly the relationship of the KMT and $g$-matrix formalisms. We will work to second order in the free $N N$ transition amplitude in the nuclear matter limit. In the $g$-matrix formalism the optical potential for the elastic scattering of a nucleon of incident momentum $k_{a}$, is expressed as the matrix elements, between antisymmetrized two nucleon states, of the $g$ matrix $g\left(\Omega_{\alpha}\right)$ evaluated at an appropriate starting energy $\Omega_{\alpha}[6]$, i.e.,

$$
U^{g}\left(k_{a}\right)=\sum_{\alpha}\left\langle\phi_{a}(0) \phi_{\alpha}(1)\left|g\left(\Omega_{\alpha}\right)\right| \phi_{a}(0) \phi_{\alpha}(1)\right\rangle_{\mathcal{A}} \text {. }
$$

We show in Appendix B that to second order in the free $N N$ amplitude and at intermediate energy this equation reduces to

$$
\begin{aligned}
U^{g}\left(k_{a}\right)= & \sum_{\alpha}\left\langle\phi_{a}(0) \phi_{\alpha}(1)\left|t_{01}^{f}\left(\Omega_{\alpha}\right)\right| \phi_{a}(0) \phi_{\alpha}(1)\right\rangle \\
& -\sum_{\alpha, \beta}\left\langle\phi_{a}(0) \phi_{\alpha}(1)\left|t_{01}^{f}\left(\Omega_{\alpha}\right)\right| \phi_{\beta}(1)\right\rangle \\
& \quad \times \frac{1}{e_{\alpha \beta}}\left\langle\phi_{\beta}(1)\left|t_{01}^{f}\left(\Omega_{\alpha}\right)\right| \phi_{a}(0) \phi_{\alpha}(1)\right\rangle
\end{aligned}
$$

with $e_{\alpha \beta}=\epsilon_{a}^{(+)}+\epsilon_{\alpha}-K_{0}-\epsilon_{\beta}, \Omega_{\alpha}=\epsilon_{a}+\epsilon_{\alpha}$, and $\epsilon_{i}$ is the kinetic energy of nucleon $i$. It is implicit in this equation, that the free $N N$ transition amplitude is antisymmetrized with respect to the interacting pair.

In the nuclear matter limit the interaction of the projectile with the target is severely restricted by momentum conservation. Momentum conservation in the nuclear matter limit restricts one to diagonal forward matrix elements of $A \Gamma$ in Eq. (19). A comparison between the diagonal matrix elements of this equation and the $g$-matrix optical potential, Eq. (21), shows that in the nuclear matter limit and at intermediate incident energies the two formalisms are essentially equivalent. Thus, as stated above, in the KMT formalism the identity of the projectile and struck nucleon is taken into account using a properly antisymmetrized $N N$ transition amplitude. The SSA term thus already includes effects due to exchange of the projectile and struck nucleon. Additionally the DSA terms modify the SSA potential to include those Pauli blocking medium effects due the identity of the struck and the other $(A-1)$ target nucleons.

As has been discussed in the literature [26], the SSA to the Watson multiple scattering expansion, which makes use of an effective interaction in which the struck nucleon is projected outside the target ground state in all intermediate states, is completely equivalent to the KMT SSA term. Like the KMT SSA therefore, the Watson SSA does not include Pauli blocking modifications to the free $N N$ amplitude due to the identity of the struck and the other $(A-1)$ target nucleons. The accurate evaluation of such terms, which enter the second-order KMT potential, is the subject of this paper.

The physical content of the second-order KMT potential is best displayed by the development that leads to Eq. (19). However, as will be discussed later in the text, there are delicate cancellations between the two components $U_{I}^{(2)}$ and $U_{I I}^{(2)}$ which need to be treated accurately to obtain a realistic estimate of the effects of the DSA terms to the optical potential. We therefore proceed to calculate the KMT potential in the form of Eqs. (9) and (10) and do not use the truncated expansion for the potential given by Eq. (18).

\section{The absorptive content}

The absorptive part of the optical potential plays an essential role in determining the elastic scattering. Tandy, Redish, and Bolle [26] made a detailed analysis of the reactive content of the SSA optical potential using unitarity relations. They concluded that the SSA predicts too much absorption [17,26]. It is important therefore to consider the mechanisms which could lead to a reduction in the absorptive content of the optical potential. In the impulse approximation to the SSA, represented in Fig. 
(a)

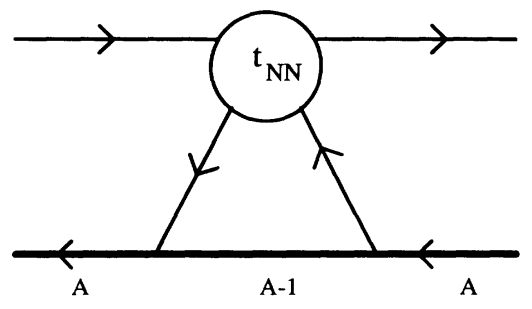

(b)

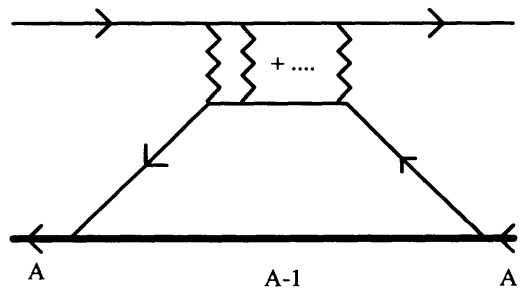

FIG. 1. (a) Diagrammatic representation of the single scattering approximation for the optical potential. (b) The $N N$ transition amplitude in (a) is open to show the intermediate states propagation of the interacting $N N$ pair.

1(a), the projectile knocks out a target nucleon, assumed free. The core of $(A-1)$ target nucleons are assumed to remain in their occupied states. In Fig. 1(b) the $N N$ transition amplitude is opened to show explicitly the intermediate states in which nucleons 0 and 1 interact as free particles with energy $E / 2$. In the intermediate state the $\boldsymbol{A}$ nucleons $(1, \ldots, \boldsymbol{A})$ are thus in a complicated linear superposition of states $\phi_{j}$ of the target, i.e.,

$$
\varphi(1,2, \ldots, A)=\sum_{j} c_{j} \phi_{j}(1,2, \ldots, A),
$$

with significant amplitudes outside the ground state. Clearly these configurations result in a loss of elastic flux. Since, in the impulse approximation, the assumption of a free struck nucleon removes all coupling with the core nucleons, the SSA generates, in some sense, the maximum loss of flux available within the KMT formalism. To reduce the absorption we expect to have to reintroduce a coupling of the struck nucleon to the core nucleons. One source of such coupling is the single particle interaction $V_{1}$. The inclusion of this term requires the solution of a three-body problem [26]. Another source of coupling is the action of the Pauli principle on the $A$ target nucleons which restricts the available phase space in the intermediate states. It is the latter effects which are the subject of the present paper.

\section{THE NN TRANSITION AMPLITUDE}

Assuming the convention of a plane wave normalized such that

$$
\langle\mathbf{r} \mid \mathbf{k}\rangle=(2 \pi)^{-3 / 2} \exp (i \mathbf{k} \cdot \mathbf{r}),
$$

the $N N$ elastic scattering amplitude for scattering from relative momentum $\mathcal{K}$ to $\mathcal{K}^{\prime}$, denoted $M\left(\omega, \mathcal{K}^{\prime}, \mathcal{K}\right)$, is re- lated to the antisymmetrized transition matrix elements by the usual relation

$$
\begin{aligned}
M\left(\omega, \mathcal{K}^{\prime}, \mathcal{K}\right) & =\left\langle\mathcal{K}^{\prime}|\boldsymbol{M}| \mathcal{K}\right\rangle \\
& =-\frac{4 \pi^{2} \mu}{\hbar^{2}}\left\langle\mathcal{K}^{\prime}\left|t_{01}^{f}(\omega)\right| \mathcal{K}\right\rangle,
\end{aligned}
$$

with $\mu$ the $N N$ reduced mass. The most general form of this amplitude, consistent with invariance under rotation, time reversal and parity is [27]

$$
\begin{aligned}
M= & \mathcal{A}+\mathcal{B}\left(\sigma_{0} \cdot \hat{\mathbf{n}}\right)\left(\sigma_{1} \cdot \hat{\mathbf{n}}\right)+\mathcal{C}\left(\sigma_{0}+\sigma_{1}\right) \cdot \hat{\mathbf{n}} \\
& +\mathcal{D}\left(\sigma_{0} \cdot \hat{\mathbf{m}}\right)\left(\sigma_{1} \cdot \hat{\mathbf{m}}\right)+\mathscr{E}\left(\sigma_{0} \cdot \hat{\mathbf{l}}\right)\left(\sigma_{1} \cdot \hat{\mathbf{l}}\right) \\
& +\mathscr{F}\left[\left(\sigma_{0} \cdot \hat{\mathbf{1}}\right)\left(\sigma_{1} \cdot \hat{\mathbf{m}}\right)+\left(\sigma_{0} \cdot \hat{\mathbf{m}}\right)\left(\sigma_{1} \cdot \hat{\mathbf{1}}\right)\right]
\end{aligned}
$$

where $\hat{\mathbf{n}}=\mathcal{K} \times \mathcal{K}^{\prime} /\left|\mathcal{K} \times \mathcal{K}^{\prime}\right|, \hat{\mathbf{l}}=\left(\mathcal{K}^{\prime}+\mathcal{K}\right) /\left|\mathcal{K}^{\prime}+\mathcal{K}\right|$, and $\hat{\mathbf{m}}=\hat{\mathbf{l}} \times \hat{\mathbf{n}}$ are the unit vectors defined by the $N N$ scattering plane. The KMT amplitudes $\mathcal{A}, \mathcal{B}, \mathcal{C}, \mathcal{D}, \mathscr{E}$, and $\mathcal{F}$ can be expressed as complex functions of the relative energy $\omega$, momentum transfer $\mathbf{q}=\left(\mathcal{K}^{\prime}-\mathcal{K}\right)$, and total momentum $Q=\left(\mathcal{K}^{\prime}+\mathcal{K}\right) / 2$ of the $N N$ pair in their center-of-mass frame. Each amplitude depends on the isotopic spin of the incident and struck nucleon in the form

$$
\mathcal{A}\left(\omega, \mathcal{K}^{\prime}, \mathcal{K}\right)=\mathcal{A}(\omega, \mathbf{q}, \boldsymbol{Q})=\mathcal{A}_{0}+\mathcal{A}_{\tau}\left(\tau_{0} \cdot \tau_{1}\right) .
$$

For ease of calculation the KMT amplitudes are expressed in terms of the decomposition of the scattering amplitude into components describing spin singlet $(S=0)$ and spin triplet $(S=1)$ scattering, $M_{v^{\prime} v}^{S}$, where $v$ and $v^{\prime}$ refer to the incident and final spin projections in the triplet state. In the representation in which these projections are referred to an axis of quantization along the incident beam direction $(\hat{\mathcal{K}})$ then

$$
\begin{aligned}
& 4 \mathcal{A}=\left(2 M_{11}^{1}+M_{00}^{1}+M_{00}^{0}\right), \\
& 4 \mathcal{B}=\left(M_{00}^{1}-2 M_{1-1}^{1}-M_{00}^{0}\right), \\
& \mathcal{C}=i\left(M_{10}^{1}-M_{01}^{1}\right) / 2 \sqrt{2},
\end{aligned}
$$

and

$$
\begin{aligned}
\mathbf{4 D}= & 2(y+2 x-1) M_{11}^{1}-\sqrt{8 x y}\left(M_{10}^{1}+M_{01}^{1}\right)+2 y M_{1-1}^{1} \\
& +(2 y-1) M_{00}^{1}-M_{00}^{0}, \\
4 \mathscr{E}= & 2(x+2 y-1) M_{11}^{1}-\sqrt{8 x y}\left(M_{10}^{1}+M_{01}^{1}\right)+2 x M_{1-1}^{1} \\
& +(2 x-1) M_{00}^{1}-M_{00}^{0}, \\
\mathcal{F}= & \sqrt{x y}\left(M_{11}^{1}-M_{00}^{0}-M_{1-1}^{1}\right)+\sqrt{2}(x-y)\left(M_{10}^{1}+M_{01}^{1}\right),
\end{aligned}
$$

where $\theta=\cos ^{-1}\left(\mathcal{K} \cdot \mathscr{K}^{\prime} /\left|\mathcal{K} \cdot \mathscr{K}^{\prime}\right|\right)$ is the $N N$ center-ofmass scattering angle. In these equations $x=\mathcal{N K}^{\prime 2} \sin ^{2} \theta$ and $y=\mathcal{N}\left(\mathcal{K}+\mathcal{K}^{\prime}\right)^{2}$ with $\mathcal{N}=1 /\left|\mathcal{K}^{\prime}+\mathcal{K}\right|^{2}$.

The amplitudes $M_{v^{\prime} v}^{S}=\left\langle\mathscr{K} S v^{\prime}|M| \mathscr{K} S v\right\rangle$ and hence the amplitudes $\mathcal{A}$ through $\mathcal{F}$, are readily obtained in terms of the partial wave components of the $N N$ amplitude, $M_{L^{\prime} L}^{J S}\left(\mathcal{K}^{\prime}, \mathcal{K}\right)$, defined according to 


$$
\begin{gathered}
\left\langle\mathcal{K}^{\prime}|\boldsymbol{M}| \mathcal{K}\right\rangle=\frac{2}{\pi} \sum_{J L L^{\prime} S M} i^{L-L^{\prime}} \mathcal{Y}_{\left(L^{\prime} S\right) J}^{M}\left(\hat{\mathcal{K}}^{\prime}\right) M_{L^{\prime} L}^{J S}\left(\mathcal{K}^{\prime}, \mathcal{K}\right) \\
\times \mathcal{Y}_{(L S) J}^{M^{\dagger}}(\hat{\mathcal{K}}),
\end{gathered}
$$

where $\mathcal{Y}_{(L S) J}^{M}$ is the spin-angle function

$$
\mathcal{Y}_{(L S) J}^{M}\left(\hat{\mathcal{K}}^{\prime}\right)=\sum_{\Lambda v}(L \Lambda S v \mid J M) Y_{L \Lambda}\left(\hat{\mathcal{K}}^{\prime}\right) X_{S v}
$$

$$
A_{0} ; q=1 \mathrm{fm}^{-1}
$$

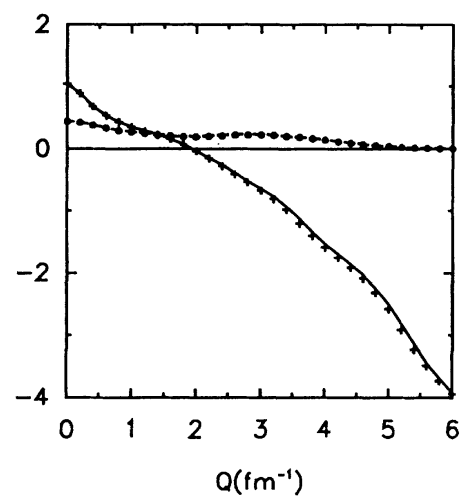

$C_{0}: q=1 \mathrm{fm}^{-1}$

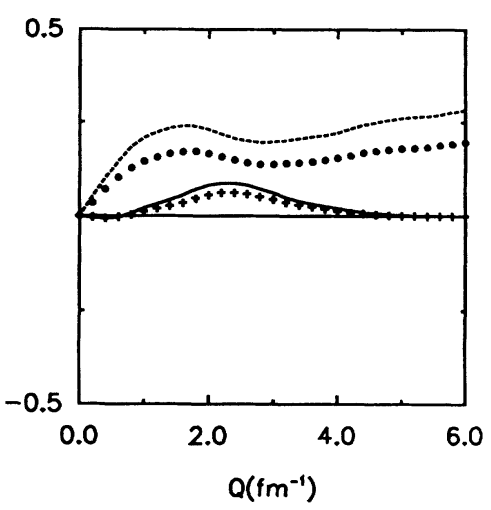

$E_{0} ; q=1 \mathrm{fm}^{-1}$

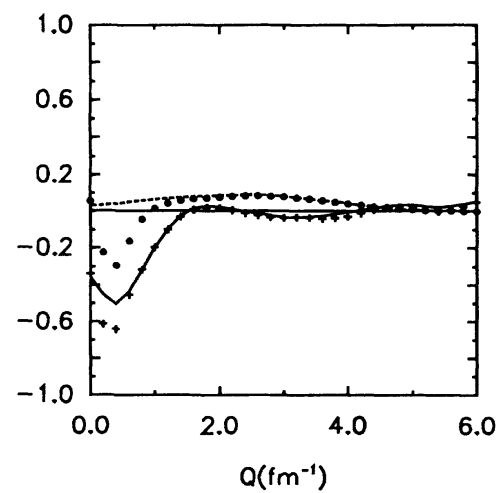

and $Y_{L \Lambda}$ and $X_{S v}$ are the spherical harmonic [28] and spin wave function of the $N N$ pair, respectively. Explicitly,

$$
\begin{aligned}
M_{v^{\prime} v}^{S}=\frac{2}{\pi} \sum_{J M L L^{\prime} \Lambda \Lambda^{\prime}} & i^{L-L^{\prime}}\left(L^{\prime} \Lambda^{\prime} S v^{\prime} \mid J M\right)(L \Lambda S v \mid J M) \\
& \times Y_{L^{\prime} \Lambda^{\prime}}\left(\hat{\mathcal{K}}^{\prime}\right) Y_{L \Lambda}^{*}(\hat{\mathcal{K}}) M_{L^{\prime} L}^{J S}\left(\mathcal{K}^{\prime}, \mathcal{K}\right) .
\end{aligned}
$$
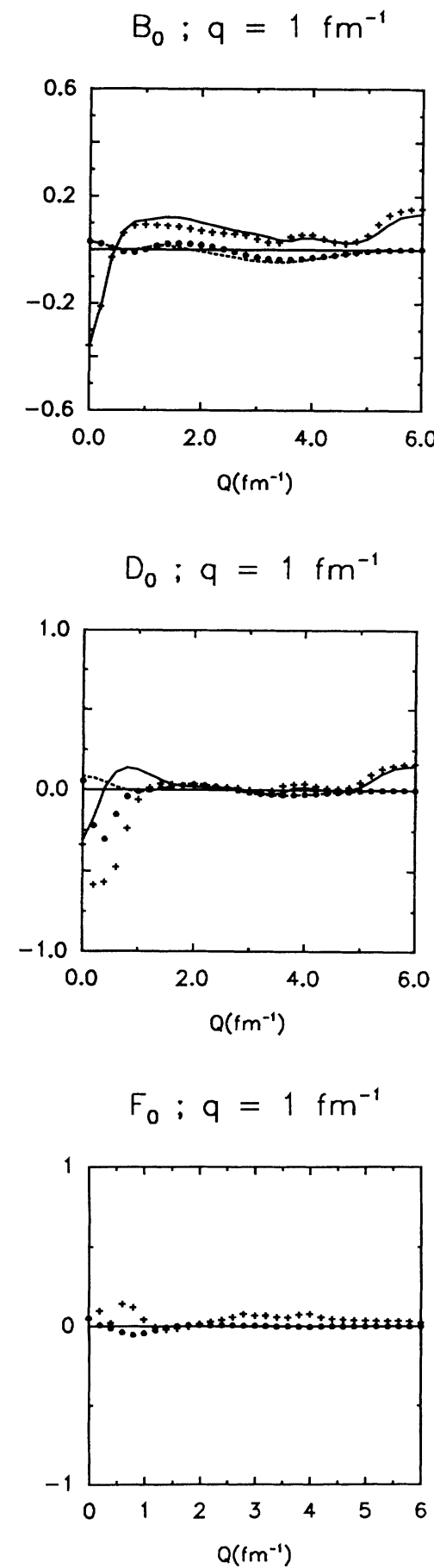

FIG. 2. Real (solid curves) and imaginary (dashed curves) parts of the isoscalar scattering amplitudes as a function of $N N$ total momentum for the Paris potential at $N N$ laboratory energy of $135 \mathrm{MeV}$. The angle $\phi$ is fixed at $\phi_{0}=\pi / 2$. The crosses and dotted points show the corresponding real and imaginary parts with $\phi=\pi / 4$. 
In the present work the free $N N$ amplitudes were calculated from the Paris potential $[15,29]$. For the evaluation of the potential matrix elements, the $N N$ amplitudes were expressed in terms of the $N N$ energy, the magnitudes of the momentum transfer and the total momentum and the angle between these two vectors $\phi$. Calculations of the off-shell isoscalar central and spin-orbit components, $\mathcal{A}_{0}$ and $\mathcal{C}_{0}$, for realistic $N N$ interactions show that, for $N N$ relative momenta less than $3 \mathrm{fm}^{-1}$ and $50 \mathrm{MeV} \leq \omega \leq 200$ $\mathrm{MeV}$, their dependence on the variables $\omega$ and $\phi$ is weak
[25]. In Fig. 2 we plot the real (solid lines) and imaginary (dashed lines) parts of the isoscalar scattering amplitudes as a function of the total momentum. The angle $\phi$ was fixed at $\phi_{0}=\pi / 2$ and the laboratory energy was $135 \mathrm{MeV}$. The plusses and solid points are the corresponding curves with angle $\phi=\pi / 4$. Figure 3 shows the corresponding isovector components. Since the optical potential for proton nucleus scattering strongly favors the diagonal components of the $N N$ amplitude we shall fix this angle to $\phi_{0}$. In this case the contribution from the amplitude $\mathcal{7}$
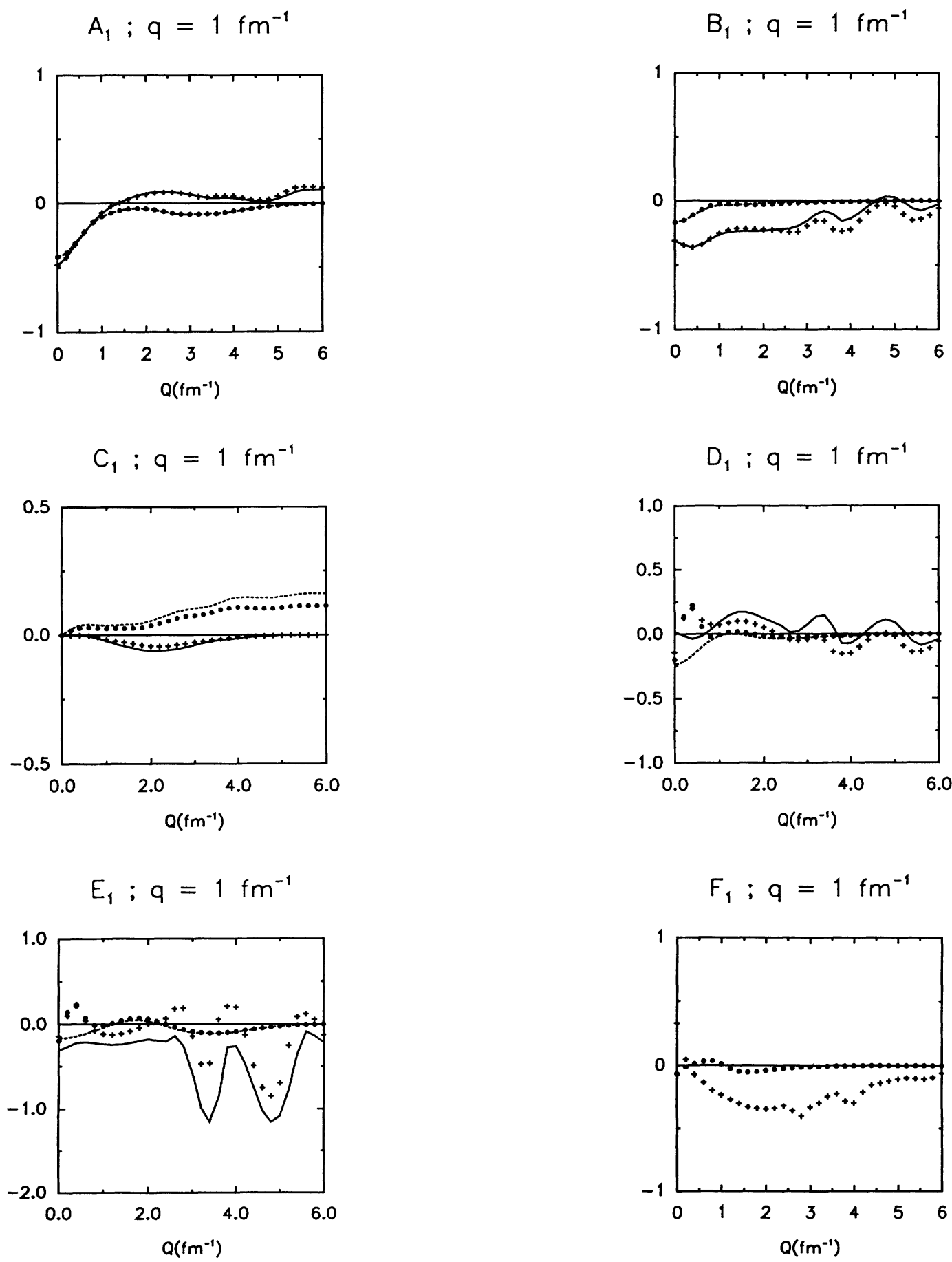

FIG. 3. The curves have the same meaning as in Fig. 2 but for the isovector components of the $N N$ scattering amplitude. 
vanishes while $\mathcal{D}$ and $\mathscr{E}$ satisfy

$$
\begin{aligned}
\mathcal{D}= & {\left[\left(M_{11}^{1}+M_{1-1}^{1}-M_{00}^{0}\right)\right.} \\
& \left.-\sec \theta\left(M_{11}^{1}-M_{1-1}^{1}-M_{00}^{0}\right)\right] / 4, \\
\mathscr{E}=[ & \left(M_{11}^{1}+M_{1-1}^{1}-M_{00}^{0}\right) \\
& \left.+\sec \theta\left(M_{11}^{1}-M_{1-1}^{1}-M_{00}^{0}\right)\right] / 4 .
\end{aligned}
$$

Detailed formulas for the required $M_{v^{\prime} v}^{S}$ amplitudes in terms of the partial wave amplitudes $M_{L^{\prime} L}^{J S}\left(\mathcal{K}^{\prime}, \mathcal{K}\right)$ for a quantization axis along the incident beam direction are collected in Appendix C.

\section{THE FINITE NUCLEUS MODEL}

The requirement of the antisymmetrization of the system of $A$ nucleons introduces a subsequent difficulty, namely, the center-of-mass motion of the target. Only $A-1$ nucleon spatial coordinates $\mathrm{r}_{i}$, referred to an arbitrary point in space, are independent as $\sum_{i} \mathbf{r}_{i} / A=\mathbf{R}$, where $\mathbf{R}$ is the center of mass of the target. To analyze the many-body problem in terms of these $(A-1)$ coordinates becomes involved, in particular because of antisymmetrization since the particles are not treated in a symmetric manner. In the shell model, the system is referred to a fixed point in space in which case the total momentum is not a constant of motion. A number of attempts have been made to treat the center-of-mass motion rigorously [30]. However, in practical calculations, such corrections are not considered beyond the harmonicoscillator model.

We consider a description of the target nucleus where a shell model single particle potential of Woods-Saxon form is assumed. In determining this shell model potential from the empirical target charge density, we do not consider center-of-mass corrections. We will also consider calculations in the case of the harmonic-oscillator (HO) shell model. For consistency with the WoodsSaxon case we will not consider center-of-mass corrections to the oscillator model. Thus, the target density distribution is evaluated directly from the HO single particle wave functions using the relative frequency obtained by Donnelly and Walker [33].

The single particle radial functions $R_{n_{\alpha} l_{\alpha} j_{\alpha}}$ are taken to be independent of the isospin of the nucleon. They are orthogonal for different $n_{\alpha}$ and are assumed normalized to unity, i.e.,

$$
\int_{0}^{\infty} d r r^{2} R_{n_{\alpha} l_{\alpha} j_{\alpha}}(r) R_{n_{\alpha}^{\prime} l_{\alpha} j_{\alpha}}(r)=\delta_{n_{\alpha} n_{\alpha}^{\prime}} .
$$

The target nucleons are assumed to move in a shell model potential, comprising central and spin-orbit nuclear interactions, in addition to the Coulomb interaction for a proton moving in the field produced by the core of the other target nucleons, i.e.,

$$
\begin{aligned}
V(r)= & V_{C}(r)-V_{0} f\left(r, R_{0}, a_{0}\right) \\
& +V_{\text {s.o. }}\left[\frac{\hbar^{2}}{m_{\pi} c}\right]^{2} \frac{1}{r} \frac{d f\left(r, R_{0}, a_{0}\right)}{d r} \mathbf{l} \cdot \boldsymbol{\sigma},
\end{aligned}
$$

with $f\left(r, R_{0}, a_{0}\right)$ the Woods-Saxon form factor

$$
\begin{aligned}
& f\left(r, R_{0}, a_{0}\right)=\left\{1+\exp \left[\left(r-R_{0}\right) / a_{0}\right]\right\}^{-1}, \\
& R_{0}=r_{0}(A-1)^{1 / 3} .
\end{aligned}
$$

The Coulomb potential is taken as due to a uniform spherical charge density with the same radius parameter as the central interaction, i.e., $r_{C}=r_{0}$.

In order to obtain a good description of the empirical charge density for ${ }^{16} \mathrm{O}$ within the single particle model we followed a similar fitting procedure to that of Elton and Swift [31]. That is, for a particular potential radius, diffuseness, and spin-orbit depth, the depths of the $S$ - and $P$-shell central interactions were adjusted to reproduce the binding energies of the $1 S_{1 / 2}$ and $1 P_{1 / 2}$ single particle
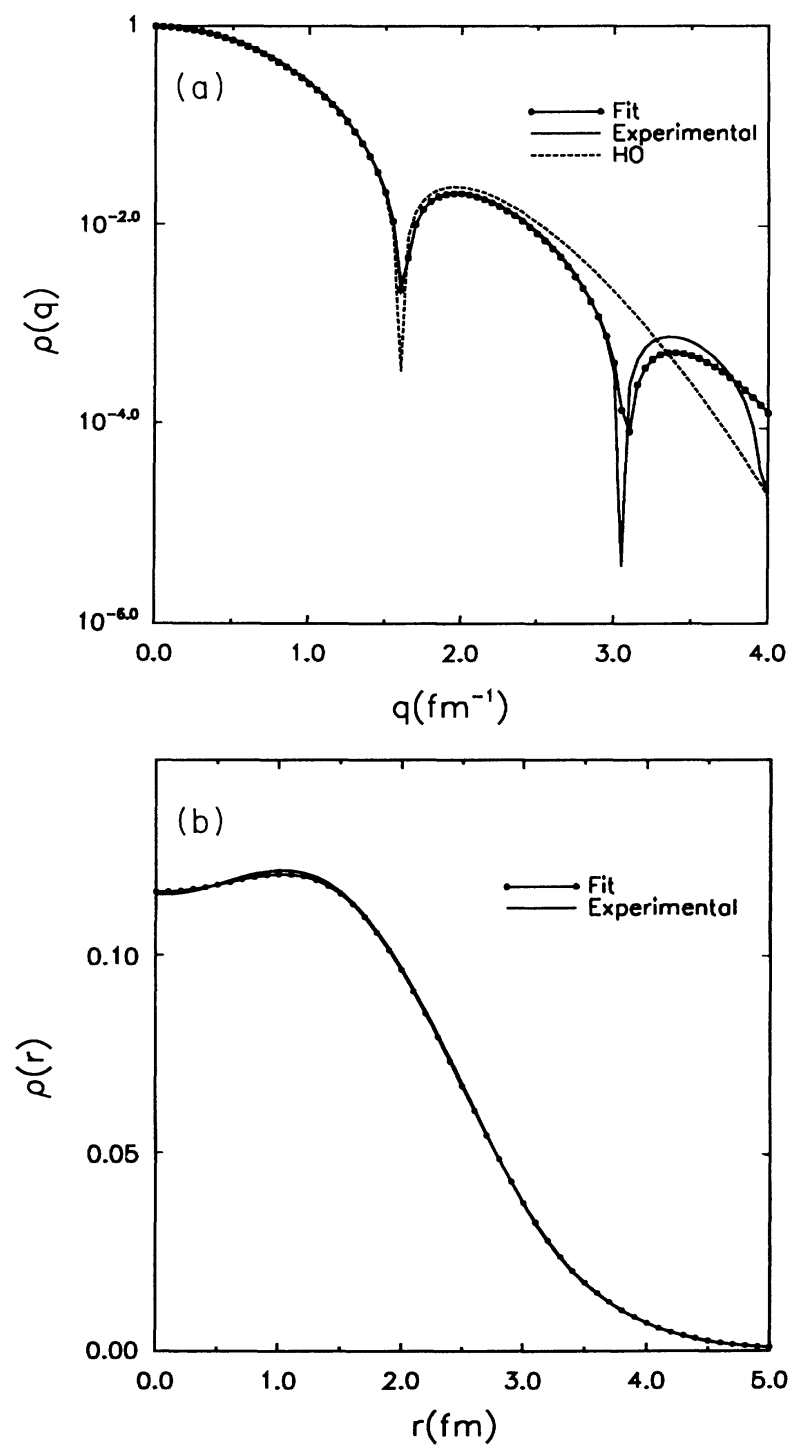

FIG. 4. The charge form factor for ${ }^{16} \mathrm{O}$ as a function of momentum transfer (a) using the modified Fermi distribution of Ref. [32] (solid line), the HO model (dashed line) and the Woods-Saxon model obtained by fitting the experimental data (solid line with dots). (b) The corresponding spatial density distributions. 
TABLE I. Woods-Saxon shell model parameters for ${ }^{16} \mathrm{O}$.

\begin{tabular}{lllccc}
\hline \hline Fit & $\begin{array}{c}V \\
(\mathrm{MeV})\end{array}$ & $\begin{array}{c}r_{0} \\
(\mathrm{fm})\end{array}$ & $\begin{array}{c}a \\
(\mathrm{fm})\end{array}$ & $\begin{array}{c}V_{\text {s.o. }} \\
(\mathrm{MeV})\end{array}$ & $\begin{array}{c}\epsilon \\
(\mathrm{MeV})\end{array}$ \\
\hline $1 P_{1 / 2}$ & 47.8 & 1.37 & 0.61 & 1.16 & 12.4 \\
$1 P_{3 / 2}$ & 47.8 & 1.37 & 0.61 & 1.16 & 13.01 \\
$1 S_{1 / 2}$ & 87.21 & 1.31 & 1.66 & & 44.0 \\
\hline \hline
\end{tabular}

states. The radial functions and hence the charge density were then calculated and a fitting routine then adjusted the radius, diffuseness, and spin-orbit depth parameters so as to reproduce the modified Fermi charge distribution of Ref. [32], which reproduces the experimental electron scattering data. In Table I we reproduce the searched values of potential parameters and in Fig. 4 we compare the calculated charge density with that of Ref. [32] and with the $\mathrm{HO}$ density [33].

When using the single particle wave functions in the optical potential calculations we do not in fact include target spin-orbit forces. The $S$ - and $P$-state single particle wave functions $R_{n_{\alpha} l_{\alpha}}(r)$ are therefore calculated as eigenfunctions of the fitted single particle potentials but in the absence of the Coulomb and spin-orbit interactions. In our fit the latter is in any case very small.

\section{THE OPTIMAL FACTORIZATION APPROXIMATION}

Assuming a single determinant for the target nucleus, the evaluation of the matrix elements of the optical potential, to any order, involves the calculation of matrix elements of the $N N$ transition operator of the general form

$$
\left\langle\mathbf{k}^{\prime}\left|\mathcal{U}_{\beta \alpha}\right| \mathbf{k}\right\rangle=\left\langle\mathbf{k}^{\prime} \beta\left|t_{01}^{f}(\omega)\right| \mathbf{k} \alpha\right\rangle .
$$

If we neglect target recoil effects and make the change of variable to $\mathbf{P}=\mathbf{p}-\mathbf{q} / 2$, we may write, using the notation of Eqs. (13) and (14),

$$
\begin{aligned}
\left\langle\mathbf{k}^{\prime}\left|\mathcal{U}_{\beta \alpha}\right| \mathbf{k}\right\rangle=\int & d \mathbf{P}\langle\beta \mid \mathbf{P}-\mathbf{q} / 2\rangle t_{01}^{f}(\omega(P), \mathbf{q}, \mathcal{Q}(P)) \\
& \times\langle\mathbf{P}+\mathbf{q} / 2 \mid \alpha\rangle,
\end{aligned}
$$

where the dependence of the $N N$ total momentum $Q$, and in the general case the $N N$ relative energy $\omega$, on $P$ is made explicit and a sum over spin and isospin indices is implicit.

In the diagonal case, $\alpha=\beta$, the product of the single particle radial wave functions is strongly peaked at $P=0$. Since the $N N$ transition amplitude is a slowly varying function of both the energy and total momentum, we introduce its Taylor expansion about $P=0$, i.e.,

$$
\begin{aligned}
t_{01}^{f}(\omega(P), \mathbf{q}, \boldsymbol{Q}(P))= & t_{01}^{f}(\omega(0), \mathbf{q}, \boldsymbol{Q}(0)) \\
& +\mathbf{P} \cdot\left[\nabla_{\mathbf{P}} t_{01}^{f}(\omega(P), \mathbf{q}, \boldsymbol{Q}(P))\right]_{P=0} \\
& +\cdots
\end{aligned}
$$

Thus, writing the required matrix elements in increasing orders in $P$,

$$
\left\langle\mathbf{k}^{\prime}\left|\mathcal{U}_{\beta \alpha}\right| \mathbf{k}\right\rangle=\left\langle\mathbf{k}^{\prime}\left|\mathcal{U}_{\beta \alpha}^{(0)}\right| \mathbf{k}\right\rangle+\left\langle\mathbf{k}^{\prime}\left|\mathcal{U}_{\beta \alpha}^{(1)}\right| \mathbf{k}\right\rangle+\cdots,
$$

where, considering only the central component of $t_{01}^{f}$ for simplicity, the zeroth-order term is

$$
\begin{aligned}
\left\langle\mathbf{k}^{\prime}\left|\mathcal{U}_{\beta \alpha}^{(0)}\right| \mathbf{k}\right\rangle= & t_{01}^{f}(\omega(0), \mathbf{q}, \boldsymbol{Q}(0)) \\
& \times \int d \mathbf{P}\langle\beta \mid \mathbf{P}-\mathbf{q} / 2\rangle\langle\mathbf{P}+\mathbf{q} / 2 \mid \alpha\rangle \\
= & t_{01}^{f}(\omega(0), \mathbf{q}, \boldsymbol{Q}(0)) \int d \mathbf{r} e^{-i \mathbf{q} \cdot \mathbf{r}}\langle\beta \mid \mathbf{r}\rangle\langle\mathbf{r} \mid \alpha\rangle
\end{aligned}
$$

and the first-order correction term is

$$
\begin{aligned}
\left\langle\mathbf{k}^{\prime}\left|\mathcal{U}_{\beta \alpha}^{(1)}\right| \mathbf{k}\right\rangle=\left[\nabla_{\mathbf{P}} t_{01}^{f}(\omega, \mathbf{q}, \mathcal{Q})\right]_{P=0} \cdot \int & d \mathbf{P} \mathbf{P}\langle\beta \mid \mathbf{P}-\mathbf{q} / 2\rangle \\
& \times\langle\mathbf{P}+\mathbf{q} / 2 \mid \alpha\rangle
\end{aligned}
$$

which thus involves integrals of the form

$$
\mathcal{J}_{\beta \alpha}(\mathbf{q})=\int d \mathbf{P} \mathbf{P}\langle\beta \mid \mathbf{P}-\mathbf{q} / 2\rangle\langle\mathbf{P}+\mathbf{q} / 2 \mid \alpha\rangle .
$$

For a closed $j$-subshell nucleus, summing over the projection $m_{\alpha}$, the integral in the last equation, and consequently the first-order correction term, vanishes in the diagonal case.

The zeroth-order term $\mathcal{U}_{\beta \alpha}^{(0)}$, with the $N N$ energy parameter $\omega(0)$ fixed at half the incident beam energy, $\omega=E / 2$, is referred to as the optimal factorization approximation. The accuracy of this approximation in the diagonal case of the first-order KMT optical potential was the subject of a number of detailed recent investigations [12-14].

\section{THE FIRST-ORDER OPTICAL POTENTIAL}

Following Ref. [14] and Eq. (8) the first-order optical potential for a closed shell isospin zero target nucleus is

$$
\begin{aligned}
\left\langle\mathbf{k}^{\prime}\left|U^{(1)}\right| \mathbf{k}\right\rangle=\frac{A-1}{A} \int & d \mathbf{P}\left\langle\mathcal{K}^{\prime}\left|\bar{t}_{01}(\varepsilon)\right| \mathcal{K}\right\rangle \\
& \times \rho(\mathbf{P}-\mathbf{q} / 2, \mathbf{P}+\mathbf{q} / 2),
\end{aligned}
$$

where $\bar{t}_{01}(\omega)$ is the spin-isospin averaged $N N$ transition matrix

$$
\left\langle\mathcal{K}^{\prime}\left|\bar{t}_{01}(\omega)\right| \mathcal{K}\right\rangle=\mathcal{A}_{0}\left(\omega, \mathcal{K}^{\prime}, \mathcal{K}\right)+\mathcal{C}_{0}\left(\omega, \mathcal{K}^{\prime}, \mathcal{K}\right) \boldsymbol{\sigma}_{0} \cdot \widehat{\mathbf{n}},
$$

with $\sigma_{0}$ the Pauli spin matrix for the projectile, $\hat{\mathbf{n}}$ the unit vector normal to the $N N$ scattering plane, and

$$
\rho(\mathbf{P}-\mathbf{q} / 2, \mathbf{P}+\mathbf{q} / 2)=4 \sum_{n_{\alpha} l_{\alpha}} \frac{\left(2 l_{\alpha}+1\right)}{4 \pi} R_{n_{\alpha} l_{\alpha}}(|\mathbf{P}-\mathbf{q} / 2|) R_{n_{\alpha} l_{\alpha}}(|\mathbf{P}+\mathbf{q} / 2|) P_{l_{\alpha}}\left(\cos \theta_{P}\right)
$$


Here $P_{l_{\alpha}}$ is the Legendre polynomial and $\theta_{P}$ the angle between the vectors $\mathbf{P}+\mathbf{q} / 2$ and $\mathbf{P}-\mathbf{q} / 2$. Expressing the matrix elements of the $N N$ transition amplitude, as above, in terms of $\mathbf{q}$ and $\mathcal{Q}$, the optimal factorization form for the single scattering potential is

$$
\left\langle\mathbf{k}^{\prime}\left|U^{(1)}\right| \mathbf{k}\right\rangle=U_{c}^{(1)}\left(\mathbf{k}^{\prime}, \mathbf{k}\right)+U_{l s}^{(1)}\left(\mathbf{k}^{\prime}, \mathbf{k}\right) \boldsymbol{\sigma}_{0} \cdot \hat{\mathbf{n}}
$$

with $\hat{\mathbf{n}}$ the unit normal to the scattering plane in the $N A$ center-of-mass frame. The central and spin-orbit interaction form factors are

$$
\begin{aligned}
& U_{c}^{(1)}\left(\mathbf{k}^{\prime}, \mathbf{k}\right)=(A-1) \mathcal{A}_{0}(\omega, \mathbf{q}, \mathbf{Q} / 2) \rho(q), \\
& U_{l s}^{(1)}\left(\mathbf{k}^{\prime}, \mathbf{k}\right)=(A-1) \mathcal{C}_{0}(\omega, \mathbf{q}, \mathbf{Q} / 2) \rho(q),
\end{aligned}
$$

where $\mathbf{Q}=\left(\mathbf{k}+\mathbf{k}^{\prime}\right) / 2$ is the mean of the incoming and outgoing projectile momenta and $\rho(q)$ is the Fourier transform of the target density normalized such that $\rho(0)=1$.

\section{THE SECOND-ORDER OPTICAL POTENTIAL}

In the evaluation of the second-order optical potential matrix elements we will assume the optimal factorization approximation, Eq. (44). Leading corrections for the evaluation of the second-order term, arising from the terms $U_{\beta \alpha}^{(1)}$ of Eq. (46), will be reported elsewhere. If, in addition, in the evaluation of the second-order potential of Eq. (9) we make the closure approximation, i.e., the single-particle energy differences $\epsilon_{\alpha}-\epsilon_{\beta}$ are replaced by an average constant, the calculation is further simplified. This constant will be taken equal to zero. We investigate the accuracy of the closure approximation in the following.

In order to assess the importance of the nonlocalities in the second-order optical potential arising from the $N N$ transition amplitude and from the intermediate state propagator, we will also consider two analogous but more approximate calculations. In the first we assume the $N N$ amplitude to take its on-shell values, leading to a local $N N$ amplitude. The second-order potential obtained in this approximation treats correctly both the nonlocality of the intermediate states propagator and the folding of the finite range and angular dependence of the $N N$ transition amplitude with the target wave function and provides an estimate of the importance of the nonlocality of the $N N$ amplitude. We also consider the yet more approximate situation where, in the second-order term, the $N N$ amplitude is fixed at its on-shell, zero momentum transfer value (the zero-range limit), which we refer to as the zero-range potential, and which provides an estimate of the importance of carrying out correctly the folding of the finite ranged $N N$ transition amplitude with the target wave function. This approximation will be expected to overestimate the second-order optical potential since we assume the maximum value of the transition amplitude for all values of the $N A$ scattering angle.

\section{A. The closure approximation}

If we neglect the single-particle energy differences $\epsilon_{\alpha}-\epsilon_{\beta}$ in Eq. (9) the momentum space matrix elements of the interaction $U_{I}^{(2)}$ can be rewritten in terms of the target correlation function [36]

$$
D\left(\mathbf{r}, \mathbf{r}^{\prime}\right)=A^{2} \rho(r) \rho\left(r^{\prime}\right)-A(A-1) \rho\left(\mathbf{r}, \mathbf{r}^{\prime}\right),
$$

where $\rho\left(\mathbf{r}, \mathbf{r}^{\prime}\right)$ is the probability density of finding a nucleon at position $\mathbf{r}$ and another at position $\mathbf{r}^{\prime}$, i.e.,

$\rho\left(\mathbf{r}, \mathbf{r}^{\prime}\right)=\frac{1}{A(A-1)}\left\langle\phi_{0}\left|\sum_{i \neq j} \delta\left(\mathbf{r}-\mathbf{r}_{i}\right) \delta\left(\mathbf{r}^{\prime}-\mathbf{r}_{j}\right)\right| \phi_{0}\right\rangle$

and $\rho(r)$ is the nuclear density normalized to unity. Thus the correlation function defined by Eq. (53) satisfies the normalization condition

$$
\frac{1}{A} \int d \mathbf{r} \int d \mathbf{r}^{\prime} D\left(\mathbf{r}, \mathbf{r}^{\prime}\right)=1
$$

The volume integral of the correlation function $D$ is therefore nonzero for a finite nucleus. Assuming a single Slater determinant for the target nucleus wave function then

$$
A(A-1) \rho\left(\mathbf{r}, \mathbf{r}^{\prime}\right)=A^{2} \rho(r) \rho\left(r^{\prime}\right)-\sum_{\alpha \beta}\left\langle\alpha(1) \beta(2)\left|\delta\left(\mathbf{r}-\mathbf{r}_{1}\right) \delta\left(\mathbf{r}^{\prime}-\mathbf{r}_{2}\right)\right| \beta(1) \alpha(2)\right\rangle
$$

and therefore

$$
D\left(\mathbf{r}, \mathbf{r}^{\prime}\right)=\sum_{\alpha \beta}\left\langle\alpha(1) \beta(2)\left|\delta\left(\mathbf{r}-\mathbf{r}_{1}\right) \delta\left(\mathbf{r}^{\prime}-\mathbf{r}_{2}\right)\right| \beta(1) \alpha(2)\right\rangle .
$$

In this form it is evident that $D\left(\mathbf{r}, \mathbf{r}^{\prime}\right)$ is generated by the exchange parts of the target wave function, i.e., due to the effects of the Pauli principle. Short-range and center-of-mass correlations which arise in a more complete description for the target wave function are not considered in the present work. The central component of $U_{I}^{(2)}$ can now be written

$$
\left\langle\mathbf{k}^{\prime}\left|U_{I}^{(2)}\right| \mathbf{k}\right\rangle=-\frac{A-1}{A} \int d \mathbf{k}^{\prime \prime} \beta\left(\omega, \mathbf{k}^{\prime}, \mathbf{k}^{\prime \prime}, \mathbf{k}\right) g\left(k^{\prime \prime}\right) \int d \mathbf{r} \int d \mathbf{r}^{\prime} e^{-i\left(\mathbf{q} \cdot \mathbf{r}+\mathbf{q}^{\prime} \cdot \mathbf{r}^{\prime}\right)} D\left(\mathbf{r}, \mathbf{r}^{\prime}\right),
$$

where $g\left(k^{\prime \prime}\right)$ is the intermediate states propagator (in momentum space representation) and $\mathbf{q}=\mathbf{k}^{\prime}-\mathbf{k}^{\prime \prime}$ and $\mathbf{q}^{\prime}=\mathbf{k}^{\prime \prime}-\mathbf{k}$ are the momentum transfers at the two $N N$ vertices. For a target of zero total spin and isospin

$$
\beta\left(\omega, \mathbf{k}^{\prime}, \mathbf{k}^{\prime \prime}, \mathbf{k}\right)=\left[\frac{\hbar^{2}}{4 \mu \pi^{2}}\right]^{2}\left\{\mathcal{A}^{2}+\left[\mathscr{B}^{2}+2 \mathcal{C}^{2}\right]\left(\hat{\mathbf{n}} \cdot \hat{\mathbf{n}}^{\prime}\right)^{2}+\mathscr{D}^{2}\left(\hat{\mathbf{m}} \cdot \hat{\mathbf{m}}^{\prime}\right)^{2}+\mathscr{E}^{2}\left(\hat{\mathbf{l}} \cdot \hat{\mathbf{l}}^{\prime}\right)^{2}\right\},
$$


where the unit vectors are defined with respect to the two $N N$ vertices, Eq. (24), and $\mathcal{A}^{2}$ through $\mathscr{E}^{2}$ are given in terms of the isoscalar and isovector components of the KMT $N N$ amplitudes, Eq. (25), according to

$$
\mathcal{A}^{2}=\mathcal{A}_{0}^{2}+3 \mathcal{A}_{\tau}^{2}
$$

We have also adopted an abbreviated notation for the squares of the components of the $N N$ amplitude, such that $\mathcal{A}_{0}^{2}=\mathcal{A}_{0}\left(\omega, \mathbf{k}^{\prime}, \mathbf{k}^{\prime \prime}\right) \mathcal{A}_{0}\left(\omega, \mathbf{k}^{\prime \prime}, \mathbf{k}\right)$, etc.

In the evaluation of Eq. (59) we make a small angle coplanar scattering approximation in treating the unit vectors. That is, we assume $\hat{\mathbf{n}} \cdot \hat{\mathbf{n}}^{\prime}=\hat{\mathbf{m}} \cdot \hat{\mathbf{m}}^{\prime}=\hat{\mathbf{l}} \cdot \hat{\mathbf{l}}^{\prime}=1$, and therefore take

$$
\begin{aligned}
\beta\left(\omega, \mathbf{k}^{\prime}, \mathbf{k}^{\prime \prime}, \mathbf{k}\right) & =\left[\frac{\hbar^{2}}{4 \mu \pi^{2}}\right]^{2}\left\{\mathcal{A}^{2}+\mathcal{B}^{2}+2 \mathscr{C}^{2}+\mathcal{D}^{2}+\mathscr{E}^{2}\right\} \\
& =\sum_{n=1}^{5}\left[\mathcal{G}^{n}\right]^{2}
\end{aligned}
$$

the index $n$ in the final equality running over the $5 N N$ amplitudes $\mathcal{A}$ to $\mathscr{E}$. Thus $\mathcal{S}^{1}=\left[\hbar^{2} / 4 \mu \pi^{2}\right] \mathcal{A}, \mathcal{G}^{3}$ $=\sqrt{2}\left[\hbar^{2} / 4 \mu \pi^{2}\right] \mathcal{C}$, etc.

The momentum space matrix elements of the term $U_{I I}^{(2)}$, Eq. (10), can similarly be written for a zero spin and isospin target as

$$
\begin{aligned}
\left\langle\mathbf{k}^{\prime}\left|U_{I I}^{(2)}\right| \mathbf{k}\right\rangle=(A-1) \int & d \mathbf{k}^{\prime \prime} \gamma\left(\omega, \mathbf{k}^{\prime}, \mathbf{k}^{\prime \prime}, \mathbf{k}\right) \\
& \times g\left(k^{\prime \prime}\right) \rho(q) \rho\left(q^{\prime}\right),
\end{aligned}
$$

where

$$
\gamma\left(\omega, \mathbf{k}^{\prime}, \mathbf{k}^{\prime \prime}, \mathbf{k}\right)=\left[\frac{\hbar^{2}}{4 \mu \pi^{2}}\right]^{2}\left\{\mathcal{A}_{0}^{2}+\mathcal{C}_{0}^{2}\left(\hat{\mathbf{n}} \cdot \hat{\mathbf{n}}^{\prime}\right)\right\} .
$$

Assuming as previously that $\hat{\mathbf{n}} \cdot \hat{\mathbf{n}}^{\prime}=1$, we have therefore

$$
\gamma\left(\omega, \mathbf{k}^{\prime}, \mathbf{k}^{\prime \prime}, \mathbf{k}\right)=\left[\frac{\hbar^{2}}{4 \mu \pi^{2}}\right]^{2}\left\{\mathcal{A}_{0}^{2}+\mathcal{C}_{0}^{2}\right\}=\sum_{n=1}^{2}\left[\mathscr{H}^{n}\right]^{2} .
$$

We note that the contributions from the dominant isoscalar central $\left(\mathcal{A}_{0}\right)$ and spin-orbit $\left(\mathcal{C}_{0}\right) N N$ components to $U_{I}^{(2)}+U_{I I}^{(2)}$ are proportional to the correlation function $C\left(\mathbf{r}, \mathbf{r}^{\prime}\right)$ of Ref. [18]. When using the HO model for the target nucleon wave functions, it can be shown [18] that the Fourier transform $C\left(\mathbf{q}, \mathbf{q}^{\prime}\right)$ vanishes for small transferred momenta, i.e., $C(\mathbf{q},-\mathbf{q}) \rightarrow 0$ when $q \rightarrow 0$. Thus, there are delicate cancellations between the two components of the KMT second-order optical potential. As the momentum transfer is increased one can expect the second-order optical potential to eventually compete with the SSA term.

We now proceed to the evaluation of the partial wave decomposition of the second-order optical potential. We first consider the term $U_{I}^{(2)}$. Assuming that the nucleus is described by a shell model wave function with no spinorbit forces, then for a closed shell nucleus

$$
\begin{aligned}
D\left(\mathbf{r}, \mathbf{r}^{\prime}\right)=\frac{1}{4 \pi^{2}} \sum_{n_{\alpha} l_{\alpha} n_{\beta} l_{\beta}} & \hat{l}_{\alpha}^{2} \widehat{l}_{\beta}^{2} R_{n_{\alpha} l_{\alpha}}(r) R_{n_{\beta} l_{\beta}}(r) \\
& \times R_{n_{\alpha} l_{\alpha}}\left(r^{\prime}\right) R_{n_{\beta} l_{\beta}}\left(r^{\prime}\right) \\
& \times \sum_{L}\left(l_{\alpha} 0 l_{\beta} 0 \mid L 0\right)^{2} P_{L}(\cos \Theta),
\end{aligned}
$$

where $\hat{l}=\sqrt{2 l+1}$ and $\Theta$ is the angle between the vectors $\mathbf{r}$ and $\mathbf{r}^{\prime}$. In the case of ${ }^{16} \mathrm{O}$, this equation reduces to

$$
\begin{aligned}
D\left(\mathbf{r}, \mathbf{r}^{\prime}\right)=\frac{1}{4 \pi^{2}}\{ & R_{10}^{2}(r) R_{10}^{2}\left(r^{\prime}\right) \\
+ & 3 R_{11}^{2}(r) R_{11}^{2}\left(r^{\prime}\right)\left[1+2 P_{2}(\cos \Theta)\right] \\
& +6 R_{10}(r) R_{11}(r) R_{10}\left(r^{\prime}\right) \\
& \left.\times R_{11}\left(r^{\prime}\right) P_{1}(\cos \Theta)\right\},
\end{aligned}
$$

or more usefully

$$
D\left(\mathbf{r}, \mathbf{r}^{\prime}\right)=\frac{6}{4 \pi^{2}} \sum_{J \leq 2, M} f_{J M}(r) f_{J M}\left(r^{\prime}\right) P_{J}(\cos \Theta)
$$

with

$$
\begin{aligned}
& f_{00}=R_{10}^{2} / \sqrt{6}, f_{01}=R_{11}^{2} / \sqrt{2}, \\
& f_{10}=R_{10} R_{11}, f_{20}=R_{11}^{2} .
\end{aligned}
$$

The partial wave decomposition of the central component of $U_{I}^{(2)}$, defined by

$$
\left\langle\mathbf{k}^{\prime}\left|U_{I}^{(2)}\right| \mathbf{k}\right\rangle=\frac{2}{\pi} \sum_{l \lambda} Y_{l \lambda}\left(\mathbf{k}^{\prime}\right) U_{I, l}^{(2)}\left(k^{\prime}, k\right) Y_{l \lambda}^{*}(\hat{\mathbf{k}}),
$$

is therefore

$$
\begin{array}{r}
U_{I, l}^{(2)}\left(k^{\prime}, k\right)=-\frac{8(A-1)}{\pi A} \sum_{n L J M} \int k^{\prime \prime 2} d k^{\prime \prime} V_{L J M}^{n, l}\left(k^{\prime}, k^{\prime \prime}\right) \\
\times g\left(k^{\prime \prime}\right) V_{L J M}^{n, l}\left(k^{\prime \prime}, k\right),
\end{array}
$$

where the potential matrix elements $V_{L J M}^{n, l}\left(k^{\prime}, k^{\prime \prime}\right)$ are

$$
\begin{gathered}
V_{L J M}^{n, l}\left(k^{\prime}, k^{\prime \prime}\right)=\sum_{l_{1} l_{2} \lambda} C_{L J}^{l_{1} l_{2} \lambda_{i} i_{2}-l_{1}} g_{l_{1} l_{2} J M}\left(k^{\prime}, k^{\prime \prime}\right) \\
\times g_{\lambda}^{n}\left(\omega, k^{\prime}, k^{\prime \prime}\right),
\end{gathered}
$$

the angular momentum coefficients are

$$
\begin{aligned}
C_{L J}^{l l_{1} l_{2} \lambda}= & {\left[\hat{\lambda}^{2} \widehat{l}_{1} \hat{l}_{2}^{2} / \hat{l}\right]\left(J 0 l_{2} 0 \mid l_{1} 0\right)\left(\lambda 0 l_{2} 0 \mid L 0\right) } \\
& \times\left(\lambda 0 l_{1} 0 \mid l 0\right) W\left(J l_{2} l \lambda ; l_{1} L\right)
\end{aligned}
$$

and the target structure enters through the radial integrals

$\mathcal{I}_{l_{1} l_{2} J M}\left(k^{\prime}, k^{\prime \prime}\right)=\int r^{2} d r j_{l_{1}}\left(k^{\prime} r\right) j_{l_{2}}\left(k^{\prime \prime} r\right) f_{J M}(r)$.

The $g_{\lambda}^{n}$ in Eq. (71) are the multipole expansion coefficients of the $n$th component of the $N N$ amplitude, defined according to

$$
g^{n}\left(\omega, \mathbf{k}^{\prime}, \mathbf{k}^{\prime \prime}\right)=\frac{2}{\pi} \sum_{\lambda \leq 6, \mu} Y_{\lambda \mu}\left(\mathbf{k}^{\prime}\right) S_{\lambda}^{n}\left(\omega, k^{\prime}, k^{\prime \prime}\right) Y_{\lambda \mu}^{*}\left(\hat{\mathbf{k}}^{\prime \prime}\right)
$$

The corresponding partial wave decomposition for $U_{I I}^{(2)}$ is

$$
\begin{array}{r}
U_{I I, l}^{(2)}\left(k^{\prime}, k\right)=\frac{2(A-1)}{\pi A} \sum_{n} \int k^{\prime \prime 2} d k^{\prime \prime} V^{n, l}\left(k^{\prime} k^{\prime \prime}\right) \\
\times g\left(k^{\prime \prime}\right) V^{n, l}\left(k^{\prime \prime}, k\right),
\end{array}
$$


but where now the potential and radial amplitudes, in terms of the multiple expansion coefficients of the $\mathscr{H}^{n}$, are $V^{n, l}\left(k^{\prime}, k^{\prime \prime}\right)=\sum_{l_{\alpha} l_{1} \lambda} C_{l l_{\alpha}}^{l_{1} \lambda} \mathcal{I}_{l_{1} l_{1} l_{\alpha} l_{\alpha}}\left(k^{\prime}, k^{\prime \prime}\right) \mathscr{H}_{\lambda}^{n}\left(\omega, k^{\prime}, k^{\prime \prime}\right)$,

$\mathcal{J}_{l_{1} l_{2} l_{\alpha} l_{\beta}}\left(k^{\prime}, k^{\prime \prime}\right)=\int r^{2} d r j_{l_{1}}\left(k^{\prime} r\right) j_{l_{2}}\left(k^{\prime \prime} r\right) R_{n_{\alpha} l_{\alpha}}(r) R_{n_{\beta} l_{\beta}}(r)$ with

$$
C_{l l_{\alpha}}^{l_{1} \lambda}=\left[\hat{l}_{\alpha} \hat{l}_{1} \hat{\lambda} / \hat{l}\right]^{2}\left(l_{1} 0 \lambda 0 \mid l 0\right)^{2}
$$

\section{B. The no closure approximation}

In the no closure approximation, in which the singleparticle energies in the propagator in Eq. (9) are retained, the partial wave decomposition of $U_{I}^{(2)}$ becomes

$$
U_{I, l}^{(2)}\left(k^{\prime}, k\right)=-\frac{8(A-1)}{\pi A} \sum_{n l_{\alpha} l_{\beta} L J} \int k^{\prime \prime 2} d k^{\prime \prime} V_{l_{\alpha} l_{\beta} L J}^{n, l}\left(k^{\prime}, k^{\prime \prime}\right) g_{l_{\alpha} l_{\beta}}\left(k^{\prime \prime}\right) \widehat{V}_{l_{\alpha} l_{\beta} L J}^{n, l}\left(k^{\prime \prime}, k\right),
$$

where

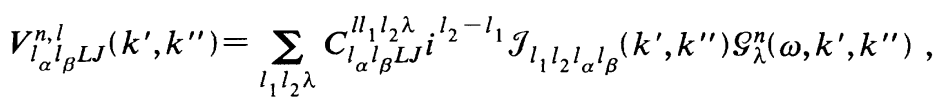

and

$$
\widehat{V}_{l_{\alpha} l_{\beta} L J}^{n, l}\left(k^{\prime \prime}, k\right)=\sum_{l_{1} l_{2} \lambda} C_{l_{\alpha} l_{\beta} L J}^{l_{1} l_{2} \lambda} i^{l_{2}-l_{1}} \mathcal{J}_{l_{2} l_{1} l_{\alpha} l_{\beta}}\left(k^{\prime \prime}, k\right) \mathcal{G}_{\lambda}^{n}\left(\omega, k^{\prime \prime}, k\right)
$$

In this case the geometric coefficients $C_{l_{\alpha} l_{\beta} L J}^{l_{1} l_{2} \lambda}$ are

$$
C_{l_{\alpha} l_{\beta} L J}^{l l_{1} l_{2} \lambda}=(-)^{l_{1}+l_{2}}\left[\hat{\lambda}^{2} \hat{l}_{\alpha} \hat{l}_{\beta} \hat{l}_{1} \hat{l}_{2} \hat{l}\right]\left(l_{\alpha} 0 l_{\beta} 0 \mid L 0\right)\left(L 0 l_{2} 0 \mid l_{1} 0\right)\left(\lambda 0 l_{1} 0 \mid l 0\right)\left(l_{2} 0 \lambda 0 \mid J 0\right) W\left(L l_{2} l \lambda ; l_{1} J\right),
$$

the integrals $\mathcal{J}_{l_{1} l_{2} l_{\alpha} l_{\beta}}\left(k^{\prime}, k^{\prime \prime}\right)$ being given by Eq. (77). The potential $U_{I I}^{(2)}$ is of course still given by the previous form, Eqs. (75) to (78).

\section{The local second-order potential}

In order to remove all nonlocalities and arrive at an entirely local expression for the second-order potential, we fix the $N N$ transition amplitude as in the zero-range case. In addition, the correlation function taken from nuclear matter is applied to the finite nucleus using the local density approximation with appropriate Fermi momentum $k_{f}=1.37 \mathrm{fm}^{-1}$. That is,

$$
\begin{aligned}
& D\left(\mathbf{r}, \mathbf{r}^{\prime}\right)=\frac{A}{4} \rho^{2}\left(\left|\mathbf{r}+\mathbf{r}^{\prime}\right| / 2\right) G_{F}^{2}\left(k_{f}\left|\mathbf{r}-\mathbf{r}^{\prime}\right|\right), \\
& G_{F}^{2}(x)=3 j_{1}(x) / x .
\end{aligned}
$$

If in addition the eikonal approximation [17,37] is now used for the intermediate state propagator and the principal value part of the propagator is neglected then we obtain the local second-order potential

$$
\begin{aligned}
& \left\langle\mathbf{k}^{\prime}\left|U^{(2)}\right| \mathbf{k}\right\rangle=i A(A-1) \frac{2 \pi^{3} \mu_{N A} R_{f}}{\hbar^{2} k_{0}} \beta_{0}(\omega) F(q), \\
& q=\left|\mathbf{k}^{\prime}-\mathbf{k}\right|,
\end{aligned}
$$

where $k_{0}$ is the on-shell entrance channel momentum and $R_{f}$ is the Fermi correlation length

$$
R_{f}=\int d x G_{F}^{2}(x)=\frac{3 \pi}{5 k_{f}}=1.38 \mathrm{fm} .
$$

$F(q)$ is the Fourier transform of the square of the target density. The quantity $\beta_{0}(\omega)$ is given by Eq. (61) but with the $N N$ amplitudes evaluated in the on-shell zero-range limit.

\section{ELASTIC SCATTERING OBSERVABLES}

To estimate the validity of the closure approximation in the intermediate state propagator we present in Fig. 5 the proton $-{ }^{16} \mathrm{O}$ elastic scattering observables at $135 \mathrm{MeV}$, calculated using the zero-range optical potential [38]. The proton target Coulomb interaction is not included. The figure shows that the closure approximation (dashed curve) provides an excellent approximation to the full (no closure) second-order optical potential (solid curve). This is in agreement with the work of Miller, Austern, and Silver [39] who showed that for a local target Hamiltonian and $N N$ amplitude, the leading corrections to the closure approximation vanish in the evaluation of the second-order term.

In order to obtain a first indication of the importance of the various nonlocalities present in the medium corrections on the absorptive nature of the optical potential we present in Fig. 6 the calculated partial wave reflection coefficients $\eta(+)$ corresponding to total angular momentum $J=L+1 / 2$. The calculations use the $\mathrm{HO}$ target wave functions. To isolate the effect of the treatment of the nonlocalities in the nuclear component of the second-order $N A$ optical potential, these calculations were performed in the absence of the nucleon-nucleus Coulomb interaction. We see from the figure that, at all energies, the nonlocal second-order calculations (solid 
curves) reduce the absorption present in the lower partial waves in comparison with the first-order KMT calculations (dotted curves with open squares). The results of calculations assuming the $N N$ transition amplitude is on the energy shell (dashed with filled triangles) show that the contribution of the nonlocality of the $N N$ amplitude
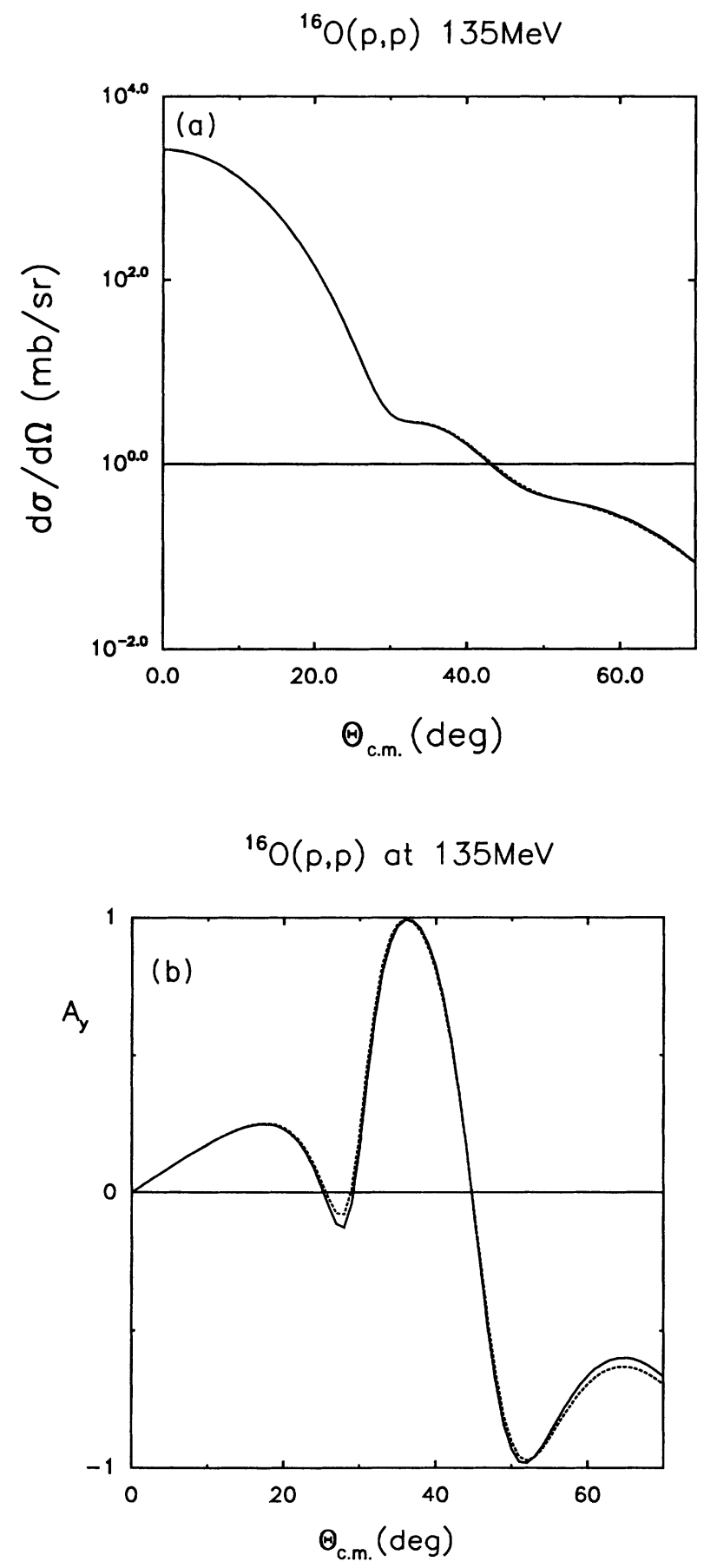

FIG. 5. (a) Calculated differential cross section and (b) analyzing power for ${ }^{16} \mathrm{O}(p, p)$ scattering at $135 \mathrm{MeV}$ using the zero-range second-order optical potential obtained without the use of closure in the intermediate state propagator (solid curve). The dashed curve is the corresponding calculation using the closure approximation. The Coulomb interaction is not included. to the second-order potential is negligible at these intermediate energies, at least within the framework of the optimal factorization approximation. This result suggests that the closure approximation still provides a good approximation in the evaluation of the second-order poten-
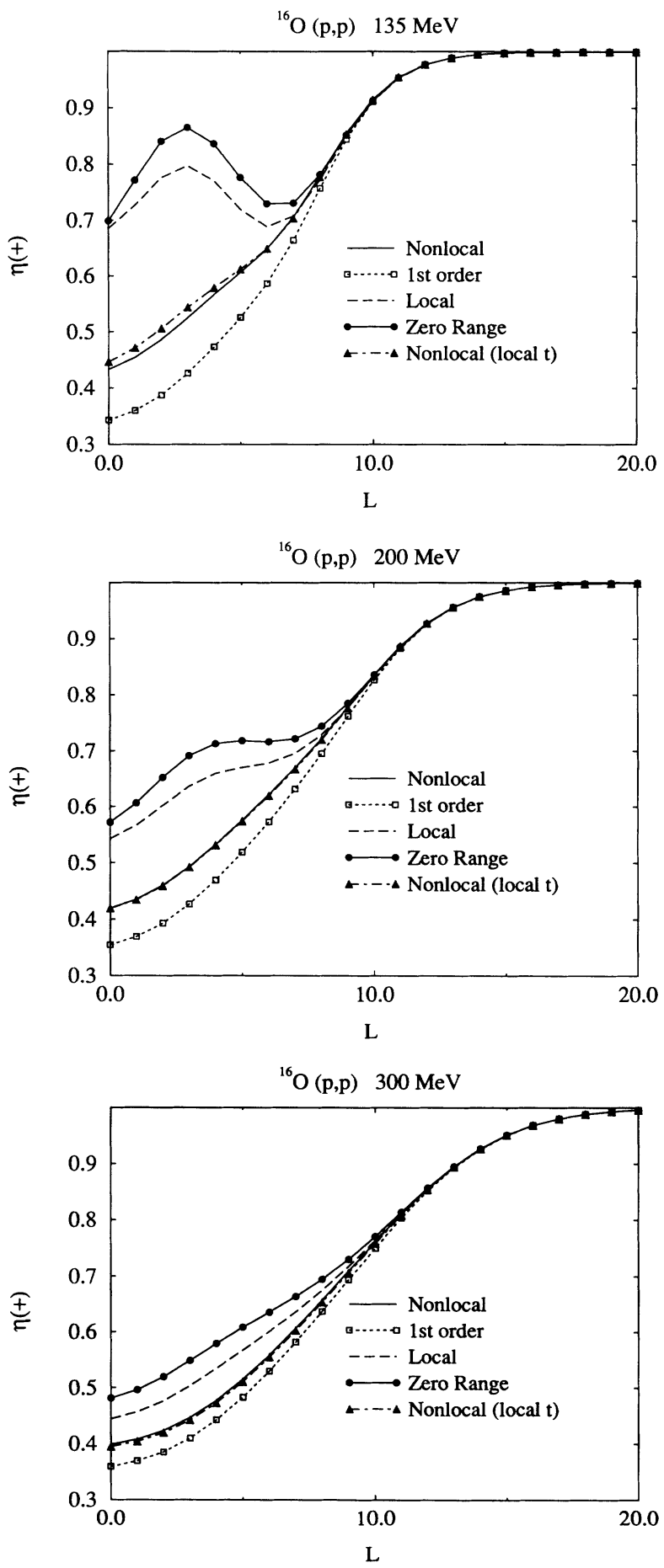

FIG. 6. Calculated reflection coefficients in the $J=L+1 / 2$ partial wave for ${ }^{16} \mathrm{O}(p, p)$ scattering at $135,200,300 \mathrm{MeV}$, predicted by the first-order (dotted with open squares) and the second-order nonlocal (solid), nonlocal with on-shell transition amplitude (dashed with filled triangles), zero-range (solid with filled circles), and local (dashed) optical potentials. 
tial which in the optimal factorization approximation probes nearly local components of the $N N$ transition amplitude. The zero-range (solid with filled circles) and local (dashed curves) calculations, on the other hand, give considerably less absorption near the grazing partial waves and make clear the need for an accurate inclusion
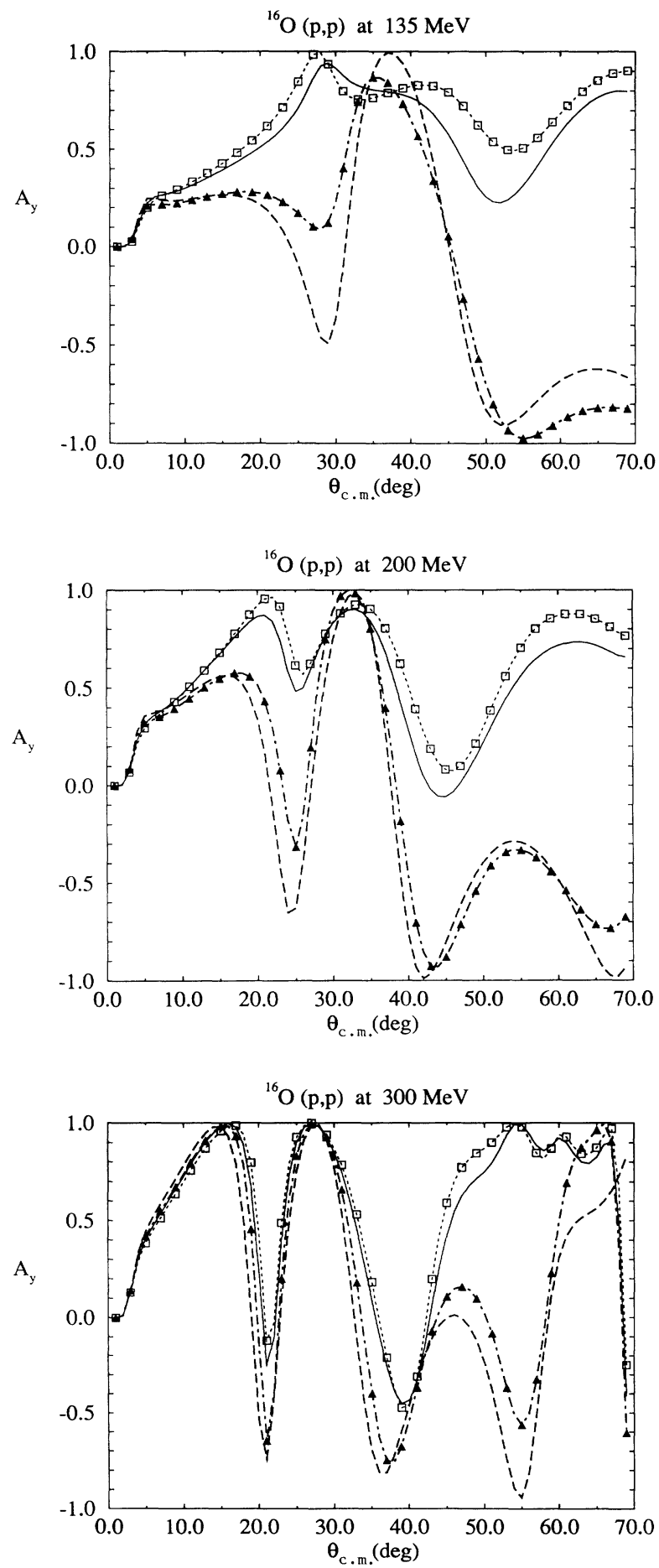

FIG. 7. Analyzing power for ${ }^{16} \mathrm{O}(p, p)$ at 135,200 , and 300 $\mathrm{MeV}$, predicted by the first-order (dotted with open squares) and the second-order nonlocal (solid), zero-range (dashed) and local (dashed with filled triangles) optical potentials. of the folding of the finite ranged $N N$ amplitude with the target wave function, within the second-order term. This effect was taken into account in an approximate way in Refs. [17-19]. The effects associated with the nonlocality of the intermediate states propagator are, by comparison, small and tend to reduce the absorption compared with the local second-order potential calculation.

Figure 7 shows the analyzing powers at the three energies under consideration which display the most sensitivity to the inclusion of the second-order effects in the deep minima. The figure shows the analyzing power for ${ }^{16} \mathrm{O}(p, p)$ at 135,200 , and $300 \mathrm{MeV}$, predicted by the first-order (dotted with open squares) and the secondorder nonlocal (solid), zero-range (dashed), and local (dashed with filled triangles) optical potentials described above. A realistic prediction of the medium effects in the polarization observables requires the evaluation of the spin-orbit contribution for the second-order optical potential. The nucleon-nucleus Coulomb potential is included in these calculations using the subtracted momentum space method [34] and assuming a uniform charge sphere density of radius $R_{c}=1.3 A^{1 / 3} \mathrm{fm}$, and a cutoff radius $R_{\text {cut }}=10 \mathrm{fm}$ [34]. The effect of the nonlocal secondorder potential contributions on the elastic cross section are small. As is evident from the figure, the introduction of the second-order medium effects does not change the qualitative angular shape of this spin-dependent observable. The zero-range (dashed) and local second-order potentials (dashed with filled triangles) by contrast, drastically overestimate the (Pauli blocking) medium effects at large angles, as might be anticipated from their use of the zero-range approximation to the $N N$ transition ampli-

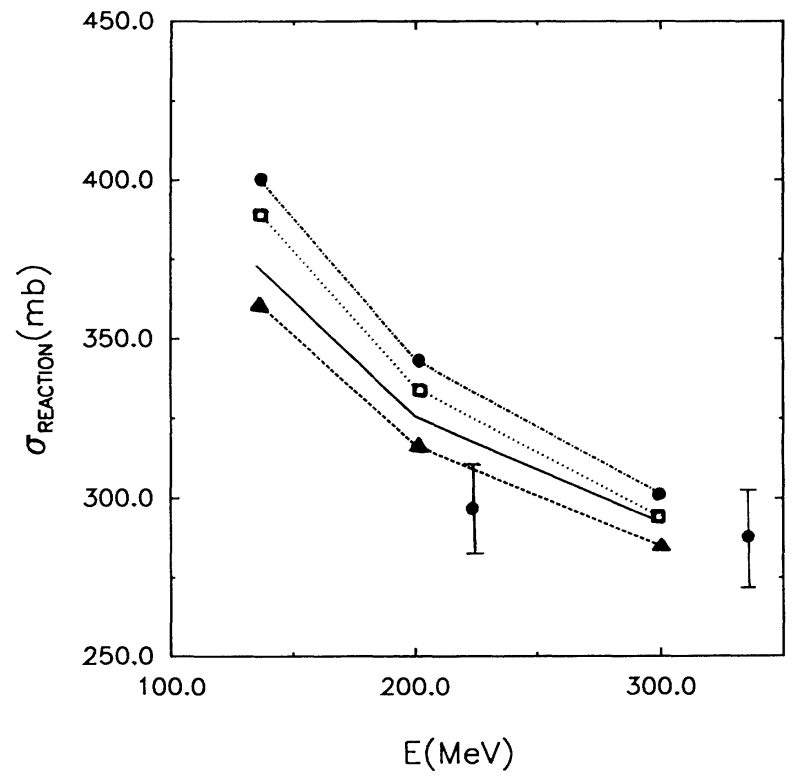

FIG. 8. Reaction cross sections for ${ }^{16} \mathrm{O}(p, p)$ as a function of the incident laboratory energy calculated using the first-order optical potential with HO (unfilled squares) and Woods-Saxon (filled circles) target wave functions. The filled triangles and solid line show the results of the second-order nonlocal calculations using the HO and WS wave functions, respectively. The data points are taken from Ref. [35]. 
tude. A similar effect on the calculated differential cross section was reported in Ref. [40].

Figure 8 shows the calculated and experimental reaction cross sections at the energies of interest. Here, the filled triangles and solid line show the results obtained from the second-order nonlocal calculations when using harmonic oscillator and Woods-Saxon wave functions, respectively. The open squares and filled circles show the corresponding calculations for the first-order potential only. The data points, with associated error bars, show the experimentally deduced values and are taken from Ref. [35]. It is evident that the nonlocal second-order potential calculations result in a significant reduction in the reaction cross sections as compared with those obtained from the first-order potential.

\section{SUMMARY AND CONCLUSIONS}

We have derived the KMT nucleon-nucleus optical potential to second order in the free $N N$ transition amplitude and shown that the second-order or double scattering term modifies the SSA to take account of Pauli blocking medium effects due to the identity of the target nucleons. The off-shell $N N$ scattering amplitudes were calculated in terms of the amplitude components describing spin singlet and spin triplet scattering and used the Paris $N N$ interaction.

The second-order nucleon optical potential was derived explicitly, in momentum space, and evaluated for proton scattering from ${ }^{16} \mathrm{O}$ at 135,200 , and $300 \mathrm{MeV}$. The calculated observables show that to obtain a realistic estimate of medium effects in nucleon-nucleus elastic scattering, we need to treat very carefully the folding of the finite range of the $N N$ transition amplitude with the target wave function. In the optimal factorization form of the second-order potential we find that the effects associated with the nonlocality of the $N N$ amplitude can be neglected at intermediate energies. The calculated medium effects result in a significant reduction in the protontarget absorption particularly in lower partial waves. Due to the surface dominance of the elastic scattering, the elastic observables are not very sensitive to such effects. Our results suggest however that medium effects generate significant modifications to proton-nucleus wave functions in low partial waves which may have implications for distorted wave calculations of nuclear reactions.

\section{ACKNOWLEDGMENTS}

The financial support of the Instituto Nacional de Investigação Científica and of the British Council (for R.C.) and of the United Kingdom Science and Engineering Research Council (SERC), in the form of Grants Nos. $\mathrm{GR} / \mathrm{F} / 4105.1$ and $\mathrm{GR} / \mathrm{F} / 1080.6$, is gratefully acknowledged. We would like also to thank Professor E. F. Redish, for providing the computer programs for the evalua- tion of the off-shell nucleon-nucleon transition matrix, Professor R. H. Landau, for the computer program LPOTP, and Dr. I. J. Thompson, for useful suggestions concerning the numerical implementation of the secondorder calculations.

\section{APPENDIX A}

In this appendix we derive expressions for the first- and second-order terms of the multiple scattering expansion of the nucleon-nucleus optical potential, Eqs. (9) and (10), assuming the target nucleus is described by a Slater determinant of occupied states. We start from Eq. (7)

$$
\begin{aligned}
U^{\mathrm{opt}}= & (A-1)\left\langle\phi_{0}\left|t_{01}(E)\right| \phi_{0}\right\rangle \\
& -(A-1)^{2}\left\langle\phi_{0}\left|t_{01}(E) \frac{P_{0}}{\delta_{0}} t_{01}(E)\right| \phi_{0}\right\rangle \\
& +\frac{A-1}{A} \sum_{i \neq j}\left\langle\phi_{0}\left|t_{0 i}(E) \frac{1}{\delta} t_{0 j}(E)\right| \phi_{0}\right\rangle .
\end{aligned}
$$

If we assume a shell model Hamiltonian

$$
H_{A}=\sum_{n=1}^{A} h_{n}, \quad h_{n}=K_{n}+V_{n}
$$

and a Slater determinant ground state wave function $\left|\phi_{0}\right\rangle$ for the target nucleus, with energy $E_{0}=0$, then the many-body propagator can be written

$$
\begin{aligned}
\frac{1}{E^{+}-K_{0}-H_{A}}= & -i \int_{0}^{\infty} d t \exp \left[i\left(E^{+}-K_{0}-H_{A}\right) t\right] \\
= & -i \int_{0}^{\infty} d t \exp \left[i\left(E^{+}-K_{0}\right) t\right] \\
& \times \exp \left[-i \sum_{n} h_{n} t\right],
\end{aligned}
$$

where we use the fact that the internal Hamiltonian of the target commutes with the incident particle kinetic energy operator, $\left[H_{A}, K_{0}\right]=0$. We will deal separately with each term in Eq. (A1).

Using the symmetry of the many-body operator $t_{01}(E)$, Eq. (4),

$$
\left\langle\phi_{0}\left|t_{01}(E)\right| \phi_{0}\right\rangle=\frac{1}{A} \sum_{n=1}^{A}\left\langle\phi_{0}\left|t_{0 n}(E)\right| \phi_{0}\right\rangle
$$

and since $t_{0 n}(E)$ satisfies

$$
\begin{aligned}
t_{0 n}(E) & =v_{0 n}+v_{0 n} \frac{1}{E^{+}-K_{0}-H_{A}} t_{0 n}(E) \\
& =v_{0 n}+v_{0 n} \frac{1}{E^{+}-K_{0}-H_{A}-v_{0 n}} v_{0 n}
\end{aligned}
$$

then, according to Eq. (A3),

$$
t_{0 n}(E)=v_{0 n}-i v_{0 n} \int_{0}^{\infty} d t \exp \left[i\left(E^{+}-K_{0}-h_{n}-v_{0 n}\right) t\right] v_{0 n} \exp \left[-i\left(H_{A}-h_{n}\right) t\right] .
$$

When acting in the target ground state clearly 


$$
\begin{aligned}
\left\langle\phi_{0}\left|t_{0 n}(E)\right| \phi_{0}\right\rangle & =\left\langle\phi_{0}\left|v_{0 n}\left[1-i \int_{0}^{\infty} d t \exp \left[i\left(E^{+}-K_{0}-h_{n}-v_{0 n}\right) t\right] v_{0 n} \exp \left[i h_{n} t\right]\right]\right| \phi_{0}\right\rangle \\
& =\frac{1}{A} \sum_{\alpha}\left\langle\alpha\left|v_{0 n}\left[1-i \int_{0}^{\infty} d t \exp \left[i\left(E^{+}+\epsilon_{\alpha}-K_{0}-h_{n}-v_{0 n}\right) t\right] v_{0 n}\right]\right| \alpha\right\rangle .
\end{aligned}
$$

Introducing the $N N$ transition operator $t_{0 n}^{f}(\omega)$

$$
t_{0 n}^{f}(\omega)=v_{0 n}+v_{0 n} \frac{1}{\omega^{+}-K} t_{0 n}^{f}(\omega)
$$

where $K$ is the kinetic energy operator for the relative motion of the active nucleon pair, and using

$$
K_{0}+K_{n}=K+\frac{\hbar^{2} \hat{\mathcal{P}}^{2}}{4 m}
$$

with $m$ the nucleon mass and $\hat{P}$ the momentum operator for the center-of-mass motion of the pair, we can therefore write

$$
\left\langle\phi_{0}\left|t_{01}(E)\right| \phi_{0}\right\rangle=\frac{1}{A} \sum_{\alpha}\left\langle\alpha\left|t_{01}^{f}\left(\hat{\omega}_{\alpha}\right)\right| \alpha\right\rangle,
$$

where the energy parameter $\widehat{\omega}_{\alpha}$ in the $N N$ transition operator is

$$
\widehat{\omega}_{\alpha}=E+\epsilon_{\alpha}-\frac{\hbar^{2} \hat{p}^{2}}{4 m}+K_{1}-h_{1}
$$

Following similar steps, the second term of Eq. (A1) is

$$
\begin{aligned}
\left\langle\phi_{0}\left|t_{01}(E) \frac{P_{0}}{\delta_{0}} t_{01}(E)\right| \phi_{0}\right\rangle=\frac{1}{A^{2}} \sum_{\alpha, \beta} & \left\langle\alpha\left|t_{01}^{f}\left(\widehat{\omega}_{\alpha}\right)\right| \alpha\right\rangle \\
& \times \frac{1}{\delta_{0}}\left\langle\beta\left|t_{01}^{f}\left(\widehat{\omega}_{\beta}\right)\right| \beta\right\rangle .
\end{aligned}
$$

To evaluate the third term we write

$$
\begin{aligned}
& \left\langle\phi_{0}\left|t_{0 i}(E) \frac{1}{\delta} t_{0 j}(E)\right| \phi_{0}\right\rangle \\
& \quad=-i \int_{0}^{\infty} d t\left\langle\phi_{0}\left|t_{0 i}(E) \exp \left[i\left(E^{+}-K_{0}\right) t\right] \mathcal{T}_{0 j}(E)\right| \phi_{0}\right\rangle,
\end{aligned}
$$

where

$$
\mathcal{T}_{0 j}(E)=\exp \left[-i h_{j} t\right] t_{0 j}(E) \exp \left[+i h_{j} t\right] .
$$

Upon defining the symmetric two-body operator

$$
\begin{aligned}
V_{i j}= & t_{0 i}(E) \exp \left[i\left(E^{+}-K_{0}\right) t\right] \mathcal{T}_{0 j}(E) \\
& +t_{0 j}(E) \exp \left[i\left(E^{+}-K_{0}\right) t\right] \mathcal{T}_{0 i}(E)
\end{aligned}
$$

then

$$
\begin{aligned}
\sum_{i \neq j}\left\langle\phi_{0}\left|t_{0 i}(E) \frac{1}{\delta} t_{0 j}(E)\right| \phi_{0}\right\rangle & =-(i / 2) \sum_{i \neq j} \int_{0}^{\infty} d t\left\langle\phi_{0}\left|V_{i j}\right| \phi_{0}\right\rangle \\
& =-(i / 2) \sum_{\alpha, \beta} \int_{0}^{\infty} d t\left[\left\langle\alpha(1) \beta(2)\left|V_{12}\right| \alpha(1) \beta(2)\right\rangle-\left\langle\alpha(1) \beta(2)\left|V_{12}\right| \beta(1) \alpha(2)\right\rangle\right]
\end{aligned}
$$

from which we obtain

$$
\begin{aligned}
\sum_{i \neq j}\left\langle\phi_{0}\left|t_{0 i}^{f}(E) \frac{1}{\delta} t_{0 j}^{f}(E)\right| \phi_{0}\right\rangle= & \sum_{\alpha, \beta}\left\langle\alpha\left|t_{01}^{f}\left(\hat{\omega}_{\alpha}\right)\right| \alpha\right\rangle \frac{1}{E^{+}-K_{0}}\left\langle\beta\left|t_{01}^{f}\left(\widehat{\omega}_{\beta}\right)\right| \beta\right\rangle \\
& -\sum_{\alpha, \beta}\left\langle\alpha\left|t_{01}^{f}\left(\hat{\omega}_{\beta}\right)\right| \beta\right\rangle \frac{1}{E^{+}-K_{0}+\epsilon_{\alpha}-\epsilon_{\beta}}\left\langle\beta\left|t_{01}^{f}\left(\widehat{\omega}_{\alpha}\right)\right| \alpha\right\rangle .
\end{aligned}
$$

Collecting these results, the $N A$ optical potential to second order in the free $N N$ transition operator is $U^{\text {opt }}=U^{(1)}+U^{(2)}$, where

$$
U^{(1)}=\frac{A-1}{A} \sum_{\alpha}\left\langle\alpha\left|t_{01}^{f}\left(\widehat{\omega}_{\alpha}\right)\right| \alpha\right\rangle,
$$

and $U^{(2)}$ is the sum of two components

$$
\begin{aligned}
U_{I}^{(2)}=-\frac{A-1}{A} \sum_{\alpha, \beta} & \left\langle\alpha\left|t_{01}^{f}\left(\widehat{\omega}_{\beta}\right)\right| \beta\right\rangle \frac{1}{E^{+}-K_{0}+\epsilon_{\alpha}-\epsilon_{\beta}} \\
& \times\left\langle\beta\left|t_{01}^{f}\left(\hat{\omega}_{\alpha}\right)\right| \alpha\right\rangle,
\end{aligned}
$$

$$
U_{I I}^{(2)}=\frac{A-1}{A^{2}} \sum_{\alpha, \beta}\left\langle\alpha\left|t_{01}^{f}\left(\widehat{\omega}_{\alpha}\right)\right| \alpha\right\rangle \frac{1}{E^{+}-K_{0}}\left\langle\beta\left|t_{01}^{f}\left(\widehat{\omega}_{\beta}\right)\right| \beta\right\rangle,
$$

where, by Eq. (A11), the energy parameter in the $N N$ transition amplitude still depends explicitly on the binding energy of the struck nucleon.

\section{APPENDIX B}

In this appendix we expand the $g$-matrix optical potential [6] to second order in the free $N N$ transition ampli- 
tude. The optical potential for the elastic scattering of a nucleon with incident momentum $k_{a}$ is

$U^{g}\left(k_{a}\right)=\sum_{\alpha} \mathcal{A}\left\langle\phi_{a}(0) \phi_{\alpha}(1)\left|g\left(\Omega_{\alpha}\right)\right| \phi_{a}(0) \phi_{\alpha}(1)\right\rangle_{\mathcal{A}}$,

where the sum in $\alpha$ runs over all occupied single particle states, $\phi_{a}$ represents the incident plane wave and the two particle states are assumed antisymmetrized, i.e.,

$\left|\phi_{a}(0) \phi_{\alpha}(1)\right\rangle_{\mathcal{A}}=\left[\left|\phi_{a}(0) \phi_{\alpha}(1)\right\rangle-\left|\phi_{a}(1) \phi_{\alpha}(0)\right\rangle\right] / \sqrt{2}$.

The $g$ matrix (for a starting energy $\Omega_{\alpha}$ ) is the solution of the integral equation

$$
g\left(\Omega_{\alpha}\right)=v+v \frac{Q_{>}}{e} g\left(\Omega_{\alpha}\right),
$$

where $v$ is the free $N N$ potential between the two nucleons and $Q_{>}$projects off the (Pauli blocked) occupied states of the target, i.e.,

$$
Q_{>}=\sum_{b<\beta \text { unocc }}|b \beta\rangle\langle b \beta| \text {. }
$$

In the propagator in Eq. (B3)

$$
e|b \beta\rangle=\left(\epsilon_{a}^{+}+\epsilon_{\alpha}-\epsilon_{b}-\epsilon_{\beta}\right)|b \beta\rangle
$$

where the starting energy is $\Omega_{\alpha}=\epsilon_{a}+\epsilon_{\alpha}$. The energies $\epsilon$ are the sum of the particle's kinetic energy and an "auxiliary potential" [6] chosen so as to improve the rate of convergence of expansion of the self-energy operator. That is,

$$
\epsilon=\hbar^{2} k^{2} / 2 m+U(\epsilon) .
$$

There is no unique prescription for the choice of this auxiliary potential, however there are a number of arguments [6] that favor the use of a self-consistent potential. Some authors take this potential to be the real part of the effective interaction [8] while others [7] assume $U(\epsilon)=0$. Here, in order to establish the relation between the $g$ matrix and the KMT multiple scattering formalism, we take this potential to be zero. The $g$ matrix and free $N N$ transition amplitude are then simply related by

$$
g\left(\Omega_{\alpha}\right)=t^{f}\left(\Omega_{\alpha}\right)+t^{f}\left(\Omega_{\alpha}\right) \frac{Q_{>}-1}{e} g\left(\Omega_{\alpha}\right) .
$$

Thus, expanding $g\left(\Omega_{\alpha}\right)$ to second order in the free $N N$ transition amplitude

$$
\begin{aligned}
{ }_{\mathcal{A}}\left\langle\phi_{a}(0) \phi_{\alpha}(1)\left|g\left(\Omega_{\alpha}\right)\right| \phi_{a}(0) \phi_{\alpha}(1)\right\rangle_{\mathcal{A}}= & { }_{\mathcal{A}}\left\langle\phi_{a}(0) \phi_{\alpha}(1)\left|t^{f}\left(\Omega_{\alpha}\right)\right| \phi_{a}(0) \phi_{\alpha}(1)\right\rangle_{\mathcal{A}} \\
& +{ }_{\mathcal{A}}\left\langle\phi_{a}(0) \phi_{\alpha}(1)\left|t^{f}\left(\Omega_{\alpha}\right) \frac{Q_{>}-1}{e} t^{f}\left(\Omega_{\alpha}\right)\right| \phi_{a}(0) \phi_{\alpha}(1)\right\rangle_{\mathcal{A}}
\end{aligned}
$$

the first term of which yields the matrix elements of the antisymmetrized free $N N$ transition amplitude

$$
{ }_{\mathcal{A}}\left\langle\phi_{a}(0) \phi_{\alpha}(1)\left|t^{f}\left(\Omega_{\alpha}\right)\right| \phi_{a}(0) \phi_{\alpha}(1)\right\rangle_{\mathcal{A}}=\left\langle\phi_{a}(0) \phi_{\alpha}(1)\left|t^{f}\left(\Omega_{\alpha}\right)\left(1-P_{01}\right)\right| \phi_{a}(0) \phi_{\alpha}(1)\right\rangle \text {. }
$$

The second term can now be written in terms of the projections on to the occupied states as

$$
\begin{aligned}
-{ }_{\mathcal{A}}\left\langle\phi_{a}(0) \phi_{\alpha}(1)\left|t^{f}\left(\Omega_{\alpha}\right) \frac{P_{<}(0)+P_{<}(1)-P_{<}(0) P_{<}(1)}{e} t^{f}\left(\Omega_{\alpha}\right)\right| \phi_{a}(0) \phi_{\alpha}(1)\right\rangle_{\mathcal{A}} \\
\quad=-\left\langle\phi_{a}(0) \phi_{\alpha}(1)\left|\left(1-P_{01}\right) t f\left(\Omega_{\alpha}\right) \frac{P_{<}(1)}{e} t^{f}\left(\Omega_{\alpha}\right)\left(1-P_{01}\right)\right| \phi_{a}(0) \phi_{\alpha}(1)\right\rangle,
\end{aligned}
$$

where the contribution from the double projection term $P_{<}(0) P_{<}(1)$, which involves an intermediate state in which there is no high-energy particles, has been neglected at these intermediate energies. To second order in the free $N N$ amplitude therefore we write $U^{g}\left(k_{a}\right)=\left\langle\phi_{a}(0)\left|U^{g}\right| \phi_{a}(0)\right\rangle$, with

$$
U^{g}=\sum_{\alpha}\left\langle\phi_{\alpha}(1)\left|t^{f}\left(\Omega_{\alpha}\right)\left(1-P_{01}\right)\right| \phi_{\alpha}(1)\right\rangle-\sum_{\alpha}\left\langle\phi_{\alpha}(1)\left|\left(1-P_{01}\right) t^{f}\left(\Omega_{\alpha}\right) \frac{P_{<}(1)}{e} t^{f}\left(\Omega_{\alpha}\right)\left(1-P_{01}\right)\right| \phi_{\alpha}(1)\right\rangle,
$$

or

$U^{g}=\sum_{\alpha}\left\langle\phi_{\alpha}(1)\left|t^{f}\left(\Omega_{\alpha}\right)\left(1-P_{01}\right)\right| \phi_{\alpha}(1)\right\rangle-\sum_{\alpha, \beta}\left\langle\phi_{\alpha}(1)\left|\left(1-P_{01}\right) t^{f}\left(\Omega_{\alpha}\right)\right| \phi_{\beta}(1)\right\rangle \frac{1}{e_{\alpha \beta}}\left\langle\phi_{\beta}(1)\left|t^{f}\left(\Omega_{\alpha}\right)\left(1-P_{01}\right)\right| \phi_{\alpha}(1)\right\rangle$,

with $e_{\alpha \beta}=\epsilon_{a}^{+}+\epsilon_{\alpha}-K_{0}-\epsilon_{\beta}$.

\section{APPENDIX C}

Since published tabulations [41] contain certain typographical errors, in this appendix we include explicit formulas for the $N N$ spin amplitudes $M_{v^{\prime} v}^{S}$ in terms of partial wave $N N$ transition amplitudes $M_{L^{\prime} L}^{J S}\left(\mathcal{K}^{\prime}, \mathcal{K}^{\prime}\right)$, Eq. (34), for an angular momentum quantization axis along the incident beam direction $\mathcal{K}^{\prime}$. We have 


$$
\begin{aligned}
& M_{00}^{0}=2 C \sum_{L=0}^{\infty} P_{L}(\cos \theta)(2 L+1) M_{L L}^{L S=0} \\
& M_{11}^{1}=C \sum_{L=0}^{\infty} P_{L}(\cos \theta)\left\{(L+2) M_{L L}^{L+1 S=1}+(2 L+1) M_{L L}^{L S=1}+(L-1) M_{L L}^{L-1 S=1}\right. \\
& \left.-[(L+1)(L+2)]^{1 / 2} M_{L L+2}^{L+1 S=1}-[(L-1) L]^{1 / 2} M_{L L-2}^{L-1 S=1}\right\}, \\
& M_{10}^{1}=\sqrt{2} C \sum_{L=1}^{\infty} P_{L}^{1}(\cos \theta)\left[M_{L L}^{L+1 S=1}-M_{L L}^{L-1 S=1}+\left(\frac{L+2}{L+1}\right]^{1 / 2} M_{L L+2}^{L+1 S=1}-\left(\frac{L-1}{L}\right)^{1 / 2} M_{L L-2}^{L-1 S=1}\right] \text {, } \\
& M_{1-1}^{1}=C \sum_{L=2}^{\infty} P_{L}^{2}(\cos \theta)\left[\frac{1}{L+1} M_{L L}^{L+1 S=1}-\frac{2 L+1}{L(L+1)} M_{L L}^{L S=1}+\frac{1}{L} M_{L L}^{L-1 S=1}\right. \\
& \left.-\left(\frac{1}{(L+1)(L+2)}\right)^{1 / 2} M_{L L+2}^{L+1 S=1}-\left(\frac{1}{(L-1) L}\right)^{1 / 2} M_{L L-2}^{L-1 S=1}\right] \text {, } \\
& M_{01}^{1}=\sqrt{2} C \sum_{L=1}^{\infty} P_{L}^{1}(\cos \theta)\left[-\frac{L+2}{L+1} M_{L L}^{L+1 S=1}+\frac{2 L+1}{L(L+1)} M_{L L}^{L S=1}\right. \\
& \left.+\frac{L-1}{L} M_{L L}^{L-1 S=1}+\left(\frac{L+2}{L+1}\right)^{1 / 2} M_{L L+2}^{L+1 S=1}-\left(\frac{L-1}{L}\right)^{1 / 2} M_{L L-2}^{L-1 S=1}\right] \text {, } \\
& M_{00}^{1}=2 C \sum_{L=0}^{\infty} P_{L}(\cos \theta)\left\{(L+1) M_{L L}^{L+1 S=1}+L M_{L L}^{L-1 S=1}+[(L+1)(L+2)]^{1 / 2} M_{L L+2}^{L+1 S=1}+[(L-1) L]^{1 / 2} M_{L L-2}^{L-1 S=1}\right\} \text {, }
\end{aligned}
$$

the sums running over all partial wave components for which $J, L$, and $L^{\prime}$ are greater than or equal to zero and that satisfy the generalized Pauli principle $(L+S+T=$ odd). The remaining amplitudes are obtained from the symmetry relation $M_{-v^{\prime}-v}^{1}=(-1)^{v-v^{\prime}} M_{v^{\prime} v^{\prime}}^{1}$. In these equations the $P_{L}^{M}(\cos \theta)$ are the associated Legendre functions,

$$
P_{L}^{M}(\cos \theta)=\sin ^{M} \theta \frac{d^{M}}{d x^{M}} P_{L}(\cos \theta), x=\cos \theta
$$

and, according to our partial wave conventions, $C=1 / 4 \pi^{2}$.

[1] E. J. Stephenson, J. Phys. Soc. Jpn. (Suppl.) 55, 316 (1985); E. J. Stephenson et al., IUCF Report, 1990.

[2] L. B. Weinstein et al., Phys. Rev. Lett. 64, 780 (1980).

[3] E. F. Redish and K. Stricker-Bauer, Phys. Rev. C 36, 513 (1987).

[4] E. F. Redish, Nucl. Phys. A463, 417c (1987).

[5] A. K. Kerman, H. McManus, and R. M. Thaler, Ann. Phys. (N.Y.) 8, 551 (1959).

[6] J. Hüfner and C. Mahaux, Ann. Phys. (N.Y.) 73, 299 (1972).

[7] F. A. Brieva and J. R. Rook, Nucl. Phys. A281, 317 (1972); H. von Geramb, in Microscopic Optical Potentials, edited by H. von Geramb (Springer Verlag, Berlin, 1987), p. 104.

[8] J. P. Jeukenne, A. Lejeune, and C. Mahaux, Phys. Rep. 25C, 83 (1976).

[9] C. Mahaux, Nucl. Phys. A396, 9c (1983).

[10] T. Cheon and E. F. Redish, Phys. Rev. C 39, 331 (1989).

[11] J. J. Kelly et al., Phys. Rev. C 39, 1222 (1989).

[12] A. F. Arellano, F. A. Brieva, and W. G. Love, Phys. Rev. C 41, 2188 (1990); A. F. Arellano, W. G. Love, and F. A. Brieva, ibid. 43, 2734 (1991).

[13] Ch. Elster, T. Cheon, E. F. Redish, and P. C. Tandy, Phys. Rev. C 41, 814 (1990); C. R. Chinn, Ch. Elster, and R. M.
Thaler, ibid. 44, 1569 (1991).

[14] R. Crespo, R. C. Johnson, and J. A. Tostevin, Phys. Rev. C 41, 2257 (1990).

[15] M. Lacombe, B. Loiseau, J. M. Richard, R. Vinh Mau, J. Côté, P. Pires, and R. de Tourreil, Phys. Rev. C 21, 861 (1980).

[16] H. Feshbach, Nucl. Phys. A518, 303 (1990).

[17] L. Ray, Phys. Rev. C 19, 1855 (1979); 20, 1857 (1979),

[18] H. Feshbach and J. Hüfner, Ann. Phys. (N.Y.) 56, 268 (1970); H. Feshbach, A. Gal, and J. Hüfner, ibid. 66, 20 (1971); E. Boridy and H. Feshbach, ibid. 109, 468 (1977).

[19] R. C. Johnson and D. C. Martin, Nucl. Phys. A192, 496 (1972); A211, 617 (1973).

[20] N. Ottenstein, E. E. van Faasen, J. A. Tjon, and S. J. Wallace, Phys. Rev. C 42, 1825 (1990); 43, 2393 (1991).

[21] K. Kaki, Nucl. Phys. A516, 603 (1990).

[22] S. J. Wallace, Annu. Rev. Nucl. Part. Sci. 37, 267 (1987).

[23] M. L. Goldberger and K. M. Watson, Collision Theory (Wiley, New York, 1964).

[24] L. Ray, Phys. Rev. C 39, 1170 (1989).

[25] E. F. Redish and K. Stricker-Bauer, Phys. Rev. C 35, 1183 (1987).

[26] P. C. Tandy, E. F. Redish, and D. Bollé, Phys. Rev. Lett. 35, 921 (1975); Phys. Rev. C 16, 1924 (1977). 
[27] L. Wolfenstein and J. Ashkin, Phys. Rev. 85, 947 (1952).

[28] D. M. Brink and G. R. Satchler, Angular Momentum (Clarendon, Oxford, 1968).

[29] An extension of the computer programs of E. F. Redish (unpublished) to calculate all of the KMT $N N$ scattering amplitudes.

[30] J. P. Elliot and T. Skyrme, Proc. R. Soc. London A232, 561 (1955); R. E. Peirls and J. Yoccoz, ibid. A70, 381 (1957); S. Gartenhaus and C. Schwartz, Phys. Rev. 108, 482 (1957); H. J. Lipkin, ibid. 110, 1395 (1958).

[31] L. R. B. Elton and A. Swift, Nucl. Phys. A94, 52 (1967).

[32] I. Sick and I. S. McCarthy, Nucl. Phys. A150, 631 (1970).

[33] T. W. Donnelly and G. E. Walker, Phys. Rev. Lett. 22, 1121 (1969).

[34] R. Crespo and J. A. Tostevin, Phys. Rev. C 41, 2615 (1990).
[35] P. U. Renberg, D. F. Measday, M. Pepin, P. Schwaller, B. Favier, and C. Richard-Serre, Nucl. Phys. A183, 81 (1972).

[36] K. W. McVoy and L. Van Hove, Phys. Rev. 125, 1034 (1962).

[37] V. Layly and R. Schaeffer, Phys. Rev. C 17, 1145 (1978).

[38] An extension of the program LPOTP [M. J. Paez, M. E. Sagen, and R. H. Landau, Comput. Phys. Commun. 52, 141 (1988)] to include the second-order components of the nucleon-nucleus optical potential.

[39] G. A. Miller, N. Austern, and M. Silver, Phys. Rev. C 17, 835 (1978).

[40] R. Crespo, Ph.D. thesis, University of Surrey, 1991; R. Crespo, R. C. Johnson, and J. A. Tostevin, Phys. Rev. C 44, R1735 (1991).

[41] M. J. Paéz and R. H. Landau, Phys. Rev. C 29, 2267 (1984). 